\title{
A DETAILED STUDY OF THE MOLECULAR AND ATOMIC GAS TOWARD THE $\gamma$-RAY SUPERNOVA REMNANT RX J1713.7-3946: SPATIAL TeV $\gamma$-RAY AND INTERSTELLAR MEDIUM GAS CORRESPONDENCE
}

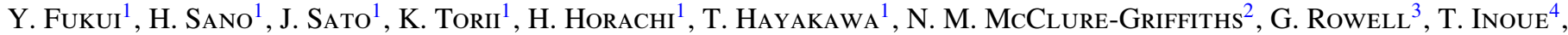 \\ S. Inutsuka $^{1}$, A. Kawamura ${ }^{1,5}$, H. Yamamoto $^{1}$, T. OKuda ${ }^{1}$, N. Mizuno ${ }^{1,5}$, T. Onishi ${ }^{1,6}$, A. Mizuno $^{7}$, And H. Ogawa ${ }^{6}$ \\ ${ }^{1}$ Department of Physics and Astrophysics, Nagoya University, Nagoya, Aichi 464-8601, Japan; fukui@ a.phys.nagoya-u.ac.jp \\ ${ }^{2}$ CSIRO Astronomy and Space Science, Epping NSW 1710, Australia \\ ${ }^{3}$ School of Chemistry and Physics, University of Adelaide, Adelaide 5005, Australia \\ ${ }^{4}$ Department of Physics and Mathematics, Aoyama Gakuin University, Sagamihara, Kanagawa 252-5258, Japan \\ ${ }^{5}$ National Astronomical Observatory of Japan, Tokyo 181-8588, Japan \\ ${ }^{6}$ Department of Astrophysics, Graduate School of Science, Osaka Prefecture University, Sakai, Osaka 599-8531, Japan \\ ${ }^{7}$ Solar-Terrestrial Environment Laboratory, Nagoya University, Nagoya, Aichi 464-8601, Japan \\ Received 2011 July 3; accepted 2011 October 13; published 2012 January 27
}

\begin{abstract}
RX J1713.7-3946 is the most remarkable TeV $\gamma$-ray supernova remnant (SNR) that emits $\gamma$-rays in the highest energy range. We have made a new combined analysis of CO and $\mathrm{H}_{\mathrm{I}}$ in the SNR and derived the total protons in the interstellar medium (ISM). We have found that the inclusion of the $\mathrm{H}$ I gas provides a significantly better spatial match between the $\mathrm{TeV} \gamma$-rays and ISM protons than the $\mathrm{H}_{2}$ gas alone. In particular, the southeastern rim of the $\gamma$-ray shell has a counterpart only in the H I. The finding shows that the ISM proton distribution is consistent with the hadronic scenario that cosmic-ray (CR) protons react with ISM protons to produce the $\gamma$-rays. This provides another step forward for the hadronic origin of the $\gamma$-rays by offering one of the necessary conditions missing in the previous hadronic interpretations. We argue that the highly inhomogeneous distribution of the ISM protons is crucial in the origin of the $\gamma$-rays. Most of the neutral gas was likely swept up by the stellar wind of an OB star prior to the supernova (SN) explosion to form a low-density cavity and a swept-up dense wall. The cavity explains the low-density site where the diffusive shock acceleration of charged particles takes place with suppressed thermal $\mathrm{X}$-rays, whereas the CR protons can reach the target protons in the wall to produce the $\gamma$-rays. The present finding allows us to estimate the total CR proton energy to be $\sim 10^{48} \mathrm{erg}, 0.1 \%$ of the total energy of the SN explosion.
\end{abstract}

Key words: cosmic rays - gamma rays: ISM - ISM: atoms - ISM: clouds - ISM: individual objects (RX J1713.7-3946) - ISM: molecules

\section{INTRODUCTION}

It is a longstanding question how cosmic-ray (CR) protons, the major constituent of CRs, are accelerated in interstellar space. Supernova remnants (SNRs) are the most likely candidates for acceleration because the high-speed shock waves offer an ideal site for diffusive shock acceleration (DSA; e.g., Bell 1978; Blandford \& Ostriker 1978). The principal site of CR proton acceleration is, however, not yet identified observationally in spite of a number of efforts to address this issue.

RX J1713.7-3946 is the brightest and most energetic TeV $\gamma$-ray SNR detected in the Galactic plane survey with H.E.S.S. (Aharonian et al. 2006a) and is a primary candidate where the origin of the $\gamma$-rays may be established. Discovery of the SNR was made in X-rays with ROSAT (Pfeffermann \& Aschenbach 1996) and ASCA showed that the X-rays are nonthermal synchrotron emission with no thermal features (Koyama et al. 1997). TeV $\gamma$-rays were first detected by CANGAROO (Enomoto et al. 2002) and, subsequently, H.E.S.S. resolved the shell-like $\mathrm{TeV} \gamma$-ray distribution with a $\sim 0.1$ point-spread function (Aharonian et al. 2004, 2006b, 2007). The $\gamma$-rays are emitted via two mechanisms, either leptonic or hadronic, closely connected to the $\mathrm{CR}$ particles and it is important to understand which mechanism is working in the SNR. The leptonic process includes the inverse Compton effect of $\mathrm{CR}$ electrons which energize the low-energy photons of the cosmic microwave background and additional soft photon fields (e.g., infrared). The hadronic process includes neutral pion decay into $\gamma$-rays following the reaction of CR protons with the low-energy target protons in the interstellar medium (ISM). Considerable work has been devoted to explaining the $\gamma$-ray emission in the framework of leptonic and hadronic scenarios (Aharonian et al. 2006b; Porter et al. 2006; Katz \& Waxman 2008; Berezhko \& Völk 2008; Ellison \& Vladimirov 2008; Tanaka et al. 2008; Morlino et al. 2009; Acero et al. 2009; Ellison et al. 2010; Patnaude et al. 2010; Zirakashvili \& Aharonian 2010; Abdo et al. 2011; Fang et al. 2011).

The molecular gas interacting with the SNR was discovered in $V_{\mathrm{LSR}}$, the velocity with respect to the local standard of rest, around $-7 \mathrm{~km} \mathrm{~s}^{-1}$ at 2.6 resolution based on the NANTEN Galactic plane CO survey, and the distance of the SNR was determined to be $1 \mathrm{kpc}$ by using the flat rotation curve of the Galaxy (Fukui et al. 2003). This determination offered a robust verification for a short distance of $1 \mathrm{kpc}$ and revised the old value of $6 \mathrm{kpc}$ derived from lower resolution $\mathrm{CO}$ observations (Slane et al. 1999). Studies of X-ray absorption suggested a similar distance of $1 \mathrm{kpc}$ under an assumption of uniform foreground gas distribution (Koyama et al. 1997), but the local bubble of Hi located by chance toward the SNR makes the X-ray absorption uncertain in estimating the distance (Slane et al. 1999; Matsunaga et al. 2001). A subsequent careful analysis of the X-ray absorption favors the shorter distance (CassamChenaï et al. 2004). At $1 \mathrm{kpc}$, the SNR has a radius of $9 \mathrm{pc}$ and an age of $1600 \mathrm{yr}$ (Fukui et al. 2003; Wang et al. 1997) and the expanding shock front has a speed of $3000 \mathrm{~km} \mathrm{~s}^{-1}$ (Zirakashvili \& Aharonian 2007; Uchiyama et al. 2003, 2007). Moriguchi et al. (2005) showed further details of the NANTEN CO distribution and confirmed the identification of the interacting 
molecular gas at -20 to $0 \mathrm{~km} \mathrm{~s}^{-1}$ by Fukui et al. (2003). Most recently, Sano et al. (2010) showed that the SNR harbors the star-forming dense cloud core named peak $\mathrm{C}$ at similar $V_{\mathrm{LSR}}$ and argued that $\mathrm{X}$-rays are bright around the core, reinforcing the association of the molecular gas.

Importantly, the associated molecular gas with the SNR opened up the possibility of identifying target protons where the hadronic process is working. If the CR density is nearly uniform, we expect that the $\gamma$-ray distribution mimics that of the interstellar target protons. Some nearby molecular clouds show good spatial correlation with relatively high-resolution $\gamma$-ray images, clearly verifying that the hadronic process is working to produce $\gamma$-rays in the CR sea (e.g., Bertsch et al. 1993 and the references therein). A detailed comparison between the ISM protons and the recent high-resolution $\gamma$-ray images of H.E.S.S. sources is useful to test the correlation, although such a test was not possible until recently in the preceding low-resolution $\gamma$-ray observations at degree-scale resolution. Aharonian et al. (2006b) compared the NANTEN CO distribution with the H.E.S.S. TeV $\gamma$-ray image and examined both leptonic and hadronic scenarios as the origin of the $\gamma$-rays. By adopting annular summing of TeV $\gamma$-rays and $\mathrm{CO}$ in the shell (see Figure 17 of Aharonian et al. 2006b), these authors found that $\mathrm{TeV} \gamma$-rays are fairly well correlated with $\mathrm{CO}$, whereas the correlation is not complete in the sense that the southeastern rim of the $\mathrm{TeV} \gamma$-ray shell has no counterpart in CO. The complete identification of target ISM protons thus remained unsettled in the hadronic scenario.

The $\gamma$-rays and X-rays are significantly enhanced toward the clumpy molecular gas at the parsec-scale as first shown by Fukui et al. (2003). A strong connection among the molecular gas, $\gamma$-rays, X-rays, and perhaps CRs is therefore suggested (Fukui et al. 2003; Fukui 2008; Sano et al. 2010; Zirakashvili $\&$ Aharonian 2010). Most of the previous models of the $\gamma$ - and X-rays cited earlier assume a more or less uniform density distribution of the ISM in the SNR, whereas the observations of the ISM indicate that the actual distribution is highly inhomogeneous, with density varying by a factor of 100 or more around the SNR. In addition, Galactic-scale studies of $\gamma$-rays suggest that there is "dark gas" which is not detectable either in $\mathrm{CO}$ or in $\mathrm{H}_{\mathrm{I}}$ but still contributes to the $\gamma$-rays and visual extinction (Grenier et al. 2005; Ade et al. 2011). Such gas may be either cold $\mathrm{H}$ I or $\mathrm{H}_{2}$ with no detectable CO. Therefore, it is important to consider $\mathrm{H}$ I carefully in order to have a comprehensive understanding of the ISM protons. In the present paper, we shall use the term "dark H I" for observed H I with significantly lower brightness than the surroundings. So, "dark H I" does not necessarily mean "dark gas" above, although they might be linked.

We present here a combined analysis of both the ${ }^{12} \mathrm{CO}$ $(J=1-0)$ and the $\mathrm{H}$ I data sets in order to clarify the distribution of the ISM protons in RX J1713.7-3946. The quantitative analysis is made mainly by using the ${ }^{12} \mathrm{CO}(J=1-0)$ and $\mathrm{H} \mathrm{I}$ data here, and a comparative study of the ${ }^{12} \mathrm{CO}(J=1-0,2-1)$ transitions is the subject of a forthcoming paper. A theoretical work of magnetohydrodynamics which incorporates fully the inhomogeneous ISM in RX J1713.7-3946 is complimentary to the present observational paper and has been published separately (Inoue et al. 2012, hereafter IYIF2012). The present paper is organized as follows. Section 2 gives the description of the $\mathrm{CO}$ and $\mathrm{H}$ I data sets. Section 3 consists of five subsections: Section 3.1 presents the $\mathrm{CO}$ and $\mathrm{H}$ I data sets; Sections 3.2-3.4 present the distribution of total ISM protons in the SNR with an emphasis on dark $\mathrm{HI}$, the cold and dense atomic phase of ISM protons; and Section 3.5 presents comparison with the $\gamma$-rays. Section 4 consists of two subsections: Section 4.1 describes the initial distribution of the ISM before the stellar explosion, and Section 4.2 the scheme of particle acceleration and its relationship with the $\gamma$-rays. The conclusions are given in Section 5.

\section{DATA SETS OF CO, H I, AND TeV $\gamma$-RAYS}

The ${ }^{12} \mathrm{CO}(J=1-0)$ data at $2.6 \mathrm{~mm}$ wavelength were taken with the NANTEN 4 m telescope in 2003 April and are identical to those published by Moriguchi et al. (2005). The system temperature of the Solid-state Imaging Spectrometer (SIS) receiver was $\sim 250 \mathrm{~K}$ in the single side band (SSB) including the atmosphere toward the zenith. The beam size of the telescope was $2^{\prime} .6$ at $115 \mathrm{GHz}$ and we adopted a grid spacing of 2'.0 in these observations. We adopt hereafter the brightness temperature $(\mathrm{K})$ as the spectral line intensity scale. The velocity resolution and rms noise fluctuations are $0.65 \mathrm{~km} \mathrm{~s}^{-1}$ and $0.3 \mathrm{~K}$, respectively.

The ${ }^{12} \mathrm{CO}(J=2-1)$ data at $1.3 \mathrm{~mm}$ wavelength were taken with the NANTEN2 $4 \mathrm{~m}$ telescope in the period from August to November in 2008 and part of the data set was published by Sano et al. (2010). The front end was a $4 \mathrm{~K}$ cooled Nb SIS mixer receiver and the SSB system temperature was $\sim 250 \mathrm{~K}$, including the atmosphere toward the zenith. The telescope had a beam size of $90^{\prime \prime}$ at $230 \mathrm{GHz}$. We used an acoustic optical spectrometers with 2048 channels having a bandwidth of $390 \mathrm{~km} \mathrm{~s}^{-1}$ and resolution per channel of $0.38 \mathrm{~km} \mathrm{~s}^{-1}$. Observations in ${ }^{12} \mathrm{CO}(J=2-1)$ were carried out in the on-the-fly mode, scanning with an integration time of 1.0-2.0 s per point. The chopper wheel method was employed for the intensity calibration and the rms noise fluctuations were better than $0.66 \mathrm{~K}$ and $0.51 \mathrm{~K}$ per channel with $1.0 \mathrm{~s}$ and $2.0 \mathrm{~s}$ integrations, respectively. An area of $2.25 \mathrm{deg}^{2}$ in a region of $346.7 \leqslant l \leqslant 348.2$ and $-1.3 \leqslant b \leqslant 0.4$ was observed.

The Hi data at $21 \mathrm{~cm}$ wavelength are from the Southern Galactic Plane Survey (McClure-Griffiths et al. 2005) and combined from the Australia Telescope Compact Array (ATCA) and the Parkes Radio Telescope. The beam size of the data set was 2.2 and we adopted a grid spacing of $40^{\prime \prime}$ toward the RX J1713.7-3946 in the current analysis. The velocity resolution and typical rms noise fluctuations were $0.82 \mathrm{~km} \mathrm{~s}^{-1}$ and $1.9 \mathrm{~K}$, respectively.

Moriguchi et al. (2005) showed an analysis of the ${ }^{12} \mathrm{CO}$ $(J=1-0)$ distribution over $100 \mathrm{~km} \mathrm{~s}^{-1}$ with a coarse velocity window of $10 \mathrm{~km} \mathrm{~s}^{-1}$ in order to test its association with the $\mathrm{SNR}$. These authors showed that the velocity range of $V_{\mathrm{LSR}}=$ -20 to $0 \mathrm{~km} \mathrm{~s}^{-1}$ has convincing signs of association with the SNR. We adopt in the present work the velocity interval -20 to $0 \mathrm{~km} \mathrm{~s}^{-1}$ for the associated ISM and present detailed ${ }^{12} \mathrm{CO}(\mathrm{J}=$ $1-0$ and 2-1) and $\mathrm{HI}$ data every $1 \mathrm{~km} \mathrm{~s}^{-1}$ (see Figure 11 in Appendix A).

For the H.E.S.S. $\gamma$-ray data, we used the combined H.E.S.S. image shown in Figure 2 of Aharonian et al. (2007). Data of 2004 and 2005 are used for this smoothed, acceptance-corrected gamma-ray excess image. The $\mathrm{TeV}$ image utilizes minimum three H.E.S.S. telescopes in event reconstruction to obtain a Gaussian standard deviation of 0.06 or FWHM of 0.14 (8'.3).

\section{COMBINED ANALYSIS OF THE CO AND H I DATA}

\subsection{Distribution of $\mathrm{CO}$ and $\mathrm{HI}$}

Figure 1(a) shows $\mathrm{TeV} \gamma$-ray distribution toward $\mathrm{RX}$ J1713.7-3946 obtained by H.E.S.S. and Figure 1(b) shows a 

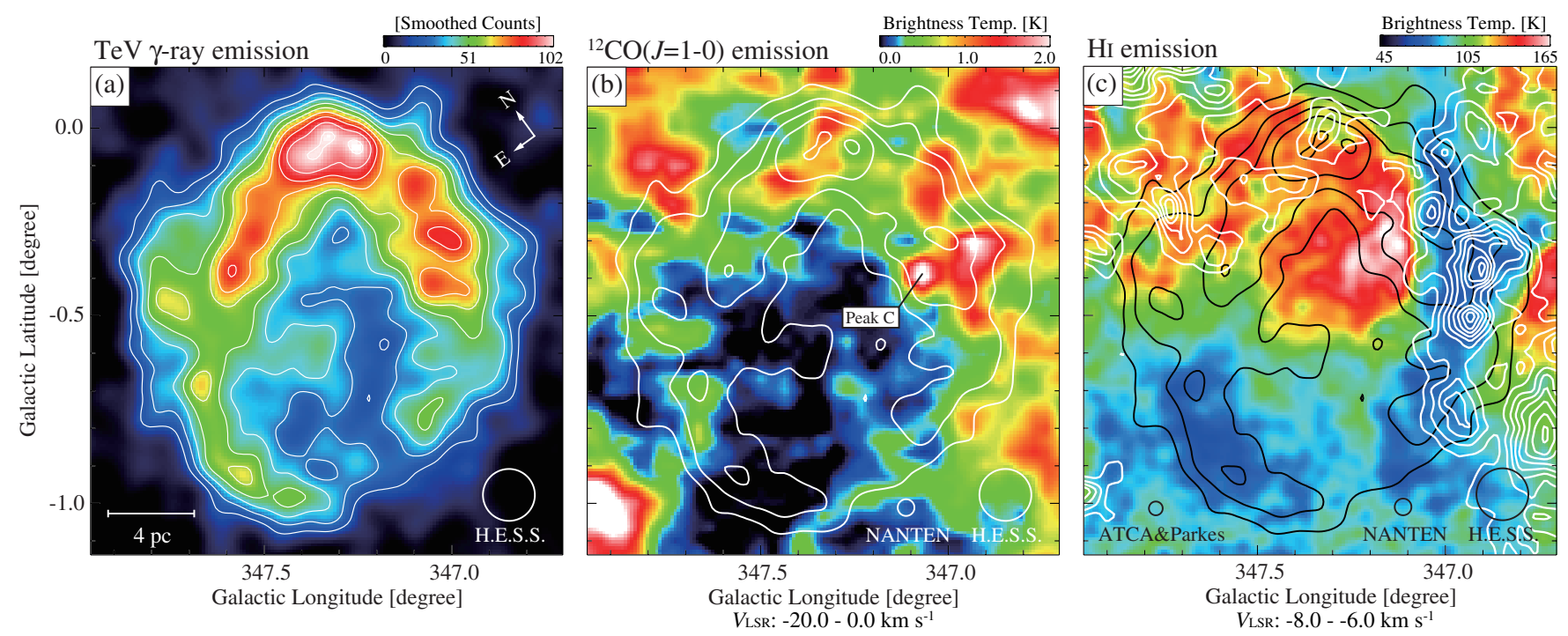

Figure 1. (a) The H.E.S.S. TeV $\gamma$-ray distribution of RX J1713.7-3946 in smoothed excess counts above the CR background (see Figure 2 of Aharonian et al. 2007). Contours are plotted every 10 smoothed counts from 20 smoothed counts. (b) Averaged brightness temperature distribution of ${ }^{12} \mathrm{CO}(J=1-0)$ emission in a velocity range of $V_{\mathrm{LSR}}=-20$ to $0 \mathrm{~km} \mathrm{~s}^{-1}$ is shown in color (Fukui et al. 2003; Moriguchi et al. 2005). White contours show the H.E.S.S. TeV $\gamma$-ray distribution and are plotted every 20 smoothed counts from 20 smoothed counts. (c) Averaged brightness temperature distribution of Hi emission obtained by ATCA and Parkes in a velocity range from $V_{\mathrm{LSR}}=-8$ to $-6 \mathrm{~km} \mathrm{~s}^{-1}$ (McClure-Griffiths et al. 2005) is shown in color. White contours show the ${ }^{12} \mathrm{CO}(J=1-0)$ brightness temperature integrated in the same velocity range every $1.0 \mathrm{~K} \mathrm{~km} \mathrm{~s}^{-1}(\sim 3 \sigma)$.
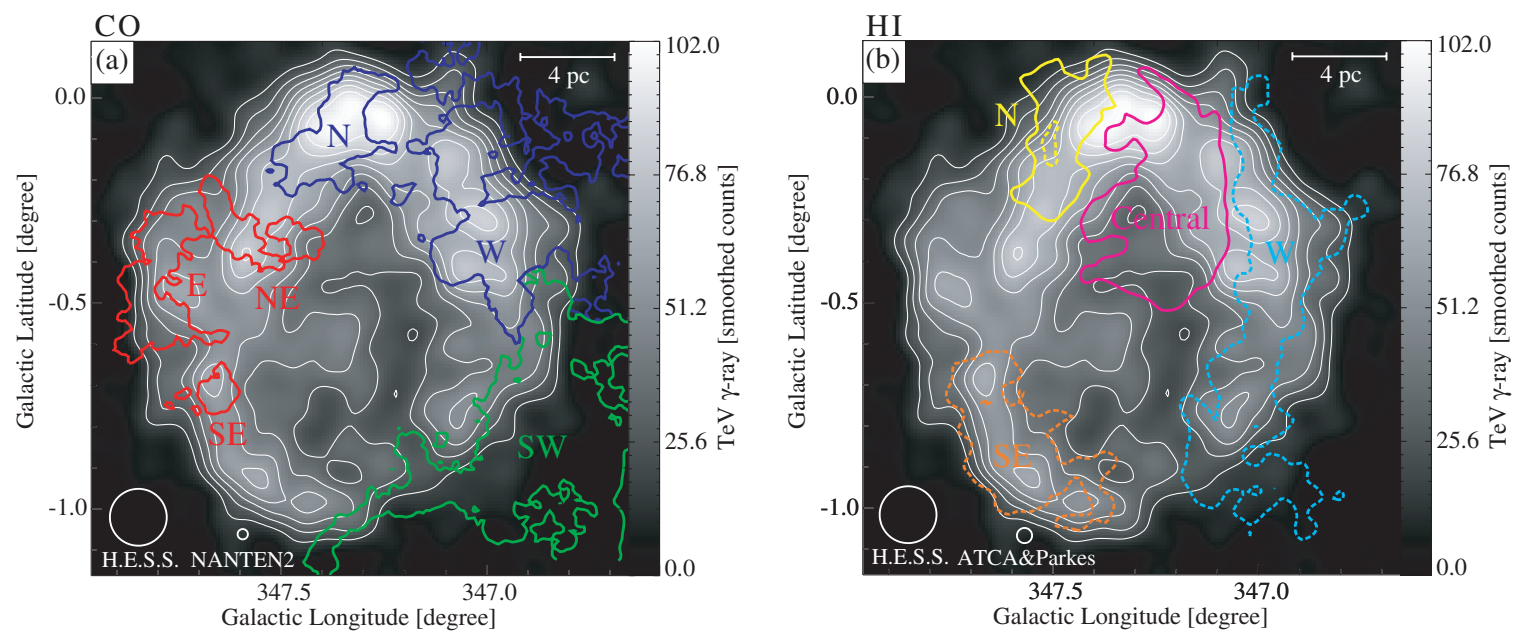

Figure 2. (a) Schematic of the identified ${ }^{12} \mathrm{CO}(J=2-1)$ clouds is shown in colored contours. The image and white contours show the TeV $\gamma$-ray distribution (Figure 1(a)). The integration velocity ranges are as follows: -8 to $-2 \mathrm{~km} \mathrm{~s}^{-1}$ (contour level: $2.3 \mathrm{~K} \mathrm{~km} \mathrm{~s}^{-1}$ ) for the $\mathrm{SW} \mathrm{cloud} \mathrm{(CO),}-17$ to $-5 \mathrm{~km} \mathrm{~s}{ }^{-1}$ (contour level: $4.9 \mathrm{~K} \mathrm{~km} \mathrm{~s}^{-1}$ ) for the $\mathrm{W}$ and $\mathrm{N}$ clouds (CO), and -6 to $0 \mathrm{~km} \mathrm{~s}^{-1}$ (contour level: $2.7 \mathrm{~K} \mathrm{~km} \mathrm{~s}^{-1}$ ) for the NE, E, and SE clouds (CO). (b) The locations of the identified $\mathrm{H}$ I clouds are shown in colored contours. The grayscale image and white contours show the TeV $\gamma$-ray distribution. The solid contours are for $\mathrm{H}$ I emission and the dashed contours are for dark $\mathrm{H}$ I. The integration velocity ranges are as follows: -8 to $-2 \mathrm{~km} \mathrm{~s}^{-1}$ (contour level: $214 \mathrm{~K} \mathrm{~km} \mathrm{~s}{ }^{-1}$ ) for the W cloud ( $\mathrm{H} \mathrm{I}$ ), -12 to $-4 \mathrm{~km} \mathrm{~s}^{-1}$ (contour level: $780 \mathrm{~K} \mathrm{~km} \mathrm{~s}^{-1}$ ) for the central and SE clouds (H I), and -14 to $-11 \mathrm{~km} \mathrm{~s}^{-1}$ (contour level: $399 \mathrm{~K} \mathrm{~km} \mathrm{~s}{ }^{-1}$ ) for the N cloud (H I).

velocity-averaged distribution of ${ }^{12} \mathrm{CO}(J=1-0)$ overlaid on the $\mathrm{TeV} \gamma$-ray distribution. The ${ }^{12} \mathrm{CO}(J=1-0)$ intensity becomes larger in the north to the Galactic plane than in the south and the most prominent features above $0.7 \mathrm{~K}$ are located in the northwest. The general ${ }^{12} \mathrm{CO}(J=1-0)$ distribution is shell-like associated with the $\gamma$-ray shell, showing weaker or no $\mathrm{CO}$ emission in part of the south. There are two regions where ${ }^{12} \mathrm{CO}(J=$ $1-0)$ delineates particularly well the outer boundary of the shell in the southwest and east. In addition, we see that some of the ${ }^{12} \mathrm{CO}(J=1-0)$ features are located within the shell including the prominent peak $\mathrm{C}$ at $(l, b)=(347.07,-0 \circ 40)$.

The ${ }^{12} \mathrm{CO}(J=2-1)$ distribution is qualitatively similar to the ${ }^{12} \operatorname{CO}(J=1-0)$ distribution. A typical ratio of the $[J=1-0] /[J=2-1]$ line intensities is $\sim 0.6$, consistent with what are derived in the other molecular clouds without heat source (e.g., Ohama et al. 2010; Torii et al. 2011). We tentatively choose three major CO clouds from Figure 11 in Appendix A, $\mathrm{W}, \mathrm{N}$, and $\mathrm{SW}$, and three minor ones, $\mathrm{E}, \mathrm{NE}$, and SE, for the sake of discussion as schematically shown in Figure 2(a), where we use ${ }^{12} \mathrm{CO}(J=2-1)$ data by taking an advantage of higher angular resolution.

Figure 1(c) shows an overlay of the $\mathrm{H}$ I distribution superposed on the ${ }^{12} \mathrm{CO}(J=1-0)$ intensity in a velocity range of -8.0 to $-6.0 \mathrm{~km} \mathrm{~s}^{-1}$. The average H I brightness temperature ranges from 45 to $150 \mathrm{~K}$ and becomes higher toward the Galactic plane. The brightest $\mathrm{HI}$ of $\sim 150 \mathrm{~K}$, the central cloud, is located toward the center of the SNR $[(l, b)=(347.25,-0.38)]$ where little ${ }^{12} \mathrm{CO}(J=1-0)$ is seen. We find dark H I clouds of around $60 \mathrm{~K}$ in the west (W cloud) and in the southeast (SE cloud). These dark H I clouds are not due to absorption of 

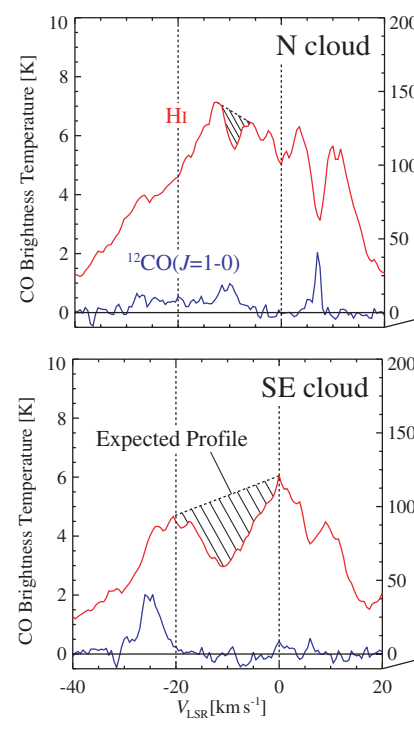

${ }^{12} \mathrm{CO}(J=1-0)$ and $\mathrm{H}$ I profiles at the four Hi clouds: the $\mathrm{N} \mathrm{cloud}(l, b)=(3470.50$

the four H clouds: the $\mathrm{N}$ cloud $(l, b)=(347.50,-0.23)$, the SE cloud $(l, b)=(347.57,-0.93)$, the central cloud

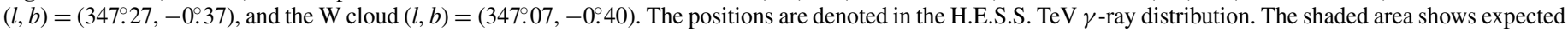
profiles behind the self-absorption.

the radio continuum radiation which is very weak toward the SNR (Lazendic et al. 2004). The dark H i W cloud corresponds well to the ${ }^{12} \mathrm{CO}(J=1-0)$ distribution, showing sharp edges both toward the east and west. The dark H I SE cloud has almost no counterpart in $\mathrm{CO}$. The relatively bright $\mathrm{HI}$ emission is seen in the north of the SNR (N cloud). The N cloud tends to be located toward ${ }^{12} \mathrm{CO}(J=1-0)$ peaks, whereas the $\mathrm{H}_{\mathrm{I}}$ brightness shows a non-monotonic, more complicated behavior than in the $\mathrm{W}$ cloud. In the northeast, we find a rim of relatively lower $\mathrm{H}$ I brightness of $\sim 100 \mathrm{~K}$ toward $(l, b)=(347.5,-0.25)$ that lies along the $\gamma$-ray shell. A schematic of the four main H i clouds is given in Figure 2(b). The good correspondence of the $\mathrm{H}$ I clouds with the $\mathrm{CO}$ and the $\gamma$-rays supports that the $\mathrm{H}_{\mathrm{I}}$ is physically associated with the SNR.

In Figure 3, we show typical $\mathrm{H}$ I and $\mathrm{CO}$ profiles in the four main $\mathrm{HI}_{\mathrm{I}}$ clouds. Figure 3 indicates that the $\mathrm{H}$ I emission is generally peaked at $-10 \mathrm{~km} \mathrm{~s}^{-1}$ with small hints of saturation, confirming that the $\mathrm{HI}$ is associated with the SNR and is generally not optically thick. We find that narrow $\mathrm{H}$ I dips having depths of 20-30 K often correspond to ${ }^{12} \mathrm{CO}(J=1-0)$ emission features in the $\mathrm{N}$ and $\mathrm{W}$ clouds. The line widths of the narrow $\mathrm{H}_{\mathrm{I}}$ dips are as small as a few $\mathrm{km} \mathrm{s}^{-1}$. It is likely that these $\mathrm{H}_{\mathrm{I}}$ dips represent residual $\mathrm{HI}$ in cold $\mathrm{CO}$ gas seen as selfabsorption. The broad H I dip in the SE cloud is also ascribed to self-absorption as argued into detail in Section 3.3. We show $\mathrm{H}_{\mathrm{I}}$ expected profiles of the background $\mathrm{HI}$ emission with a straight-line approximation as dashed areas in Figure 3 (e.g., Sato \& Fukui 1978).

\subsection{Molecular Protons}

In order to convert the ${ }^{12} \mathrm{CO}(J=1-0)$ intensity into the total molecular column density, we use an $X$ factor, which is defined as $X\left(\mathrm{~cm}^{-2}\left(\mathrm{~K} \mathrm{~km} \mathrm{~s}^{-1}\right)^{-1}\right)=N\left(\mathrm{H}_{2}\right)\left(\mathrm{cm}^{-2}\right) / W\left({ }^{12} \mathrm{CO}\right)\left(\mathrm{K} \mathrm{km} \mathrm{s}^{-1}\right)$. In order to derive an $X$ factor, the ${ }^{12} \mathrm{CO}(J=1-0)$ intensity is compared with the cloud dynamical mass (virial mass), or with the $\gamma$-rays produced via the interaction of CR protons with molecular clouds. An $X$ factor therefore accounts for the total hadronic mass and is observationally uniform in the Galactic disk (e.g., Fukui \& Kawamura 2010). We here adopt an $X$ factor of $2.0 \times 10^{20} W\left({ }^{12} \mathrm{CO}\right)\left(\mathrm{cm}^{-2}\left(\mathrm{~K} \mathrm{~km} \mathrm{~s}^{-1}\right)^{-1}\right)$ derived from the $\gamma$-rays and ${ }^{12} \mathrm{CO}(J=1-0)$ intensity in the Galaxy (Bertsch et al. 1993). We double the $\mathrm{H}_{2}$ column density to derive the ISM protons in molecular form as shown in Figure 7(a). Compared with the ${ }^{12} \mathrm{CO}(J=1-0)$ line, the ${ }^{12} \mathrm{CO}(J=2-1)$ line is not a common probe of the molecular mass. This is in part because the ${ }^{12} \mathrm{CO}(J=2-1)$ emission samples a smaller portion, having a higher excitation condition, of a molecular cloud than traced by the ${ }^{12} \mathrm{CO}(J=1-0)$ emission. We estimate for instance that a typical fraction in area of the ${ }^{12} \mathrm{CO}(J=2-1)$ emission to the ${ }^{12} \mathrm{CO}(J=1-0)$ emission is about $70 \%-80 \%$ at the half-intensity level convolved to the same beam size in the present region from the $\mathrm{CO}$ data in Figure 11 in Appendix A.

\subsection{Atomic Protons \\ 3.3.1. Optically Thin Case}

We use the $21 \mathrm{~cm} \mathrm{H}$ I transition to estimate the atomic proton column density. A usual assumption is that the $\mathrm{HI}$ emission is optically thin and the following relationship is used to calculate the $\mathrm{H}$ i column density:

$$
N_{\mathrm{p}}(\mathrm{HI})=1.823 \times 10^{18} \int T_{L}(V) d V\left(\mathrm{~cm}^{-2}\right),
$$

where $T_{L}(V)$ is the observed Hi brightness temperature $(\mathrm{K})$ (Dickey \& Lockman 1990). We note that this simple assumption is usually valid and apply Equation (1) to the regions where no $\mathrm{H}$ I dips are seen. It is certain that the narrow $\mathrm{H}$ I dips in the $\mathrm{W}$ and $\mathrm{N}$ clouds represent self-absorption by cold residual $\mathrm{H} \mathrm{I}$ in $\mathrm{CO}$ gas from their exact coincidence with $\mathrm{CO}$ in velocity. The most prominent dark H I cloud, the SE cloud, shows large line widths, which are not very common as self-absorption features. We shall examine if the SE cloud represents self-absorption below.

\subsubsection{The Dark H I SE Cloud}

We first show the integrated intensity image of the SE cloud in Figure 4(a). The H I contours are at every $3.9 \sigma$ noise level 

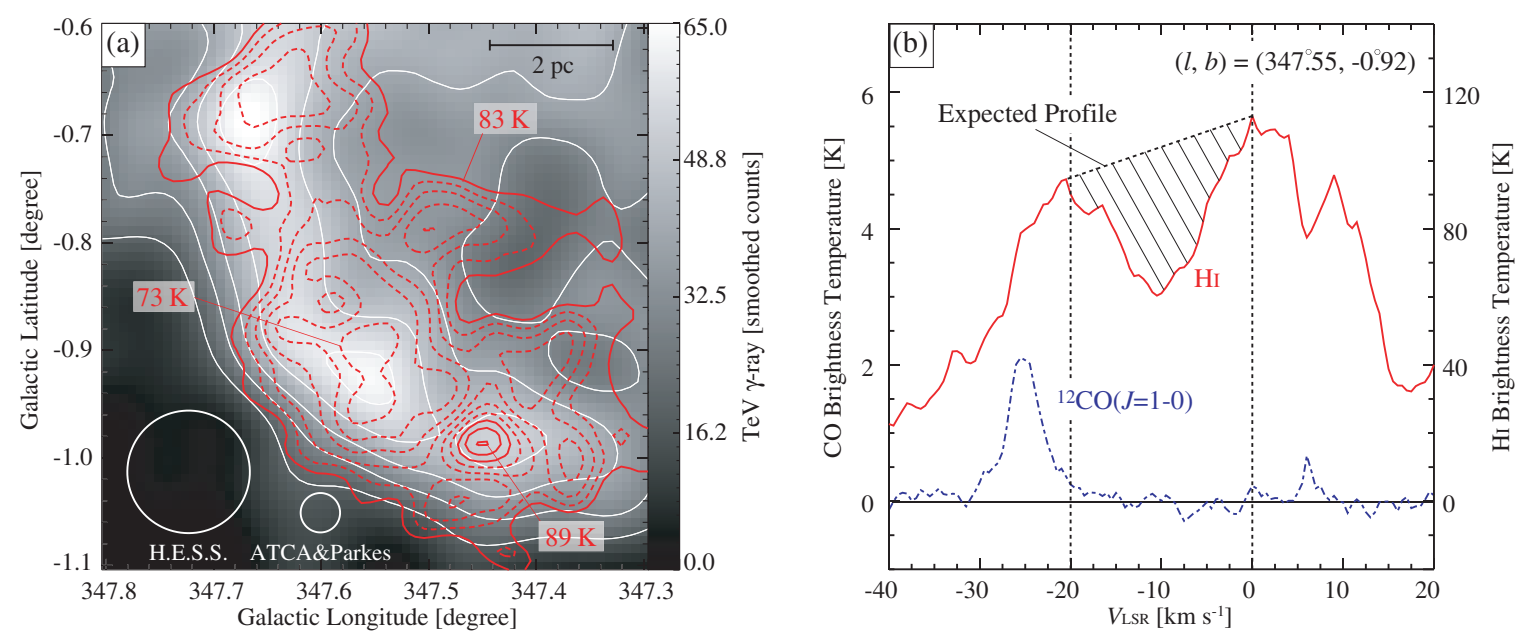

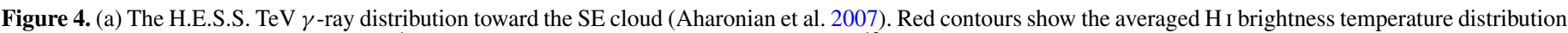

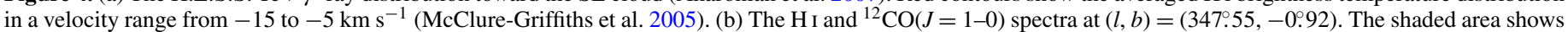
an expected $\mathrm{H}$ i profile.

and show significant details not apparent in Figure 1(c), where a coarser color code is used. We find that the Hi brightness variation is generally well correlated with the shell of $\gamma$-rays in gray scale in the sense that $\mathrm{H}$ I brightness decreases toward the enhanced $\gamma$-rays. This trend lends a support for the physical connection of the SE cloud with the $\gamma$-ray shell and may be interpreted as due to a decrease in spin temperature with a density increase in the self-absorbing H I gas (see Section 3.3.3). No ${ }^{12} \mathrm{CO}(J=1-0)$ emission is seen toward the SE cloud, expect for a possible small counterpart at $(l, b)=(347.64,-0.72)$ and $V_{\mathrm{LSR}}=-6$ to $0 \mathrm{~km} \mathrm{~s}^{-1}$ (Figures 2(a) and 11), suggesting that the density of the SE cloud is lower than that of the CO clouds.

Figure 4(b) shows a typical H I profile in the SE cloud having a deep and broad dip. The large velocity span of $20 \mathrm{~km} \mathrm{~s}^{-1}$ is not very common as a self-absorption feature; in nearby dark clouds $\mathrm{H}$ i self-absorption is generally narrow with a few $\mathrm{km} \mathrm{s}^{-1}$ in line width (e.g., Krčo \& Goldsmith 2010), whereas H I selfabsorption as broad as $10 \mathrm{~km} \mathrm{~s}^{-1}$ is seen in giant molecular clouds (e.g., Sato \& Fukui 1978). The SE cloud delineates the $\gamma$-ray shell (Figure 4(a)) and is possibly compressed gas by the wind of a high-mass star, the SN progenitor. We have investigated the velocity distribution of the SE cloud as given in Appendix A. We find that the SE cloud shows a strong velocity gradient which matches the blueshifted part of an expanding swept-up shell. Such a shell is a natural outcome of the stellarwind compression by the SN progenitor, supporting that the broad $\mathrm{HI}_{\mathrm{I}}$ dip is ascribed to the acceleration of H I gas by the wind. A H I stellar-wind shell in Pegasus driven by an early B star indeed shows a line width as large as $15 \mathrm{~km} \mathrm{~s}^{-1}$ (Section 4.1; Yamamoto et al. 2006), similar to that of the SE cloud. The difference from the narrow $\mathrm{H}$ I dips in the $\mathrm{W}$ and $\mathrm{N}$ clouds may be due to density; the SE cloud has lower density and is subject to stronger acceleration than the $\mathrm{CO}$ clouds with narrow $\mathrm{H}$ I dips (see for further discussion Section 4.1), whereas the CO clouds having higher density are less accelerated by the wind, making a systematic velocity gradient less clear in $\mathrm{CO}$ than in $\mathrm{HI}$ (see Figure 14 in Appendix B).

Figure 5(a) shows the distribution of the extinction $A_{\mathrm{V}}$ toward RX J1713.7-3946 (Dobashi et al. 2005), and indicates that the SE cloud, as well as the rest of the shell, is traced by the enhanced optical extinction. This lends another support for the self-absorption interpretation of the SE cloud. Figure 5(b) shows the total (molecular and atomic) ISM proton column density both in the SNR (derived later in Section 3.4) and in the foreground within $1 \mathrm{kpc}$, which is supposed to correspond mainly to the optical extinction. The total proton column density $N_{\mathrm{p}}$ of $\sim 10^{22} \mathrm{~cm}^{-2}$ in Figure 5(b) corresponds to an extinction of $\sim 4$ mag if we adopt the relationship $N_{\mathrm{p}}\left(\mathrm{cm}^{-2}\right)=2.5 \times 10^{21}$. $A_{\mathrm{V}}$ (magnitude) (Jenkins \& Savage 1974). The extinction toward the SE cloud is 2-3 mag in Figure 5(a) and is consistent with the H I self-absorption by considering the contamination by the foreground stars which tends to reduce $A_{\mathrm{V}}$ toward the Galactic plane.

In summary, we find it a reasonable interpretation that the SE cloud represents H I self-absorption associated with the SNR shell.

\subsubsection{Analysis of the H I Self-absorption Dips}

We shall briefly review some basic properties of $\mathrm{H}$ I gas in order to understand the behavior of Hi brightness (e.g., Sato $\&$ Fukui 1978). The spin temperature, $T_{\mathrm{s}}$, of $\mathrm{H}_{\mathrm{I}}$ is $\sim 100 \mathrm{~K}$ or higher in warm neutral medium at particle density less than $10 \mathrm{~cm}^{-3} \cdot T_{\mathrm{s}}$ decreases with density from $100 \mathrm{~K}$ down to $10 \mathrm{~K}$ in a density range of $100-1000 \mathrm{~cm}^{-3}$ (e.g., Figure 2 in Goldsmith et al. 2007). The temperature decrease is mainly due to higher shielding of stellar radiation and increased line cooling.

It is well established that $\mathrm{HI}_{\mathrm{I}}$ is converted into $\mathrm{H}_{2}$ on dust surfaces with increasing gas column density and UV shielding, and that $\mathrm{H}_{2}$ is dissociated by CRs and UV photons (e.g., Allen \& Robinson 1977). The equilibrium H I abundance is determined by the balance between formation and destruction of $\mathrm{H}_{2}$, and the residual density of $\mathrm{H}_{\mathrm{I}}$ is about $10^{-2}$ that of $\mathrm{H}_{2}$ in typical interstellar molecular clouds (Allen \& Robinson 1977; Sato \& Fukui 1978). We also note that the $\mathrm{H}_{2}$ abundance should be time dependent since the formation of $\mathrm{H}_{2}$ is a slow process on the order of $10 \mathrm{Myr}$ for density around $100 \mathrm{~cm}^{-3}$ (e.g., Allen \& Robinson 1977).

Based on the $\mathrm{HI}-\mathrm{H}_{2}$ transition, we interpret the dark $\mathrm{H}_{\mathrm{I}}$ in Figure 2(b) as representing the $\mathrm{HI}$ with lower $T_{\mathrm{s}}$. The CO W cloud shows a good spatial coincidence with the dark $\mathrm{H}_{\mathrm{I}}$ $\mathrm{W}$ cloud as is consistent with the interpretation. The other prominent dark $\mathrm{H}$ I region, the $\mathrm{SE}$ cloud, shows no $\mathrm{CO}$, and we suggest that its density is lower and its $T_{\mathrm{s}}$ is higher than that 

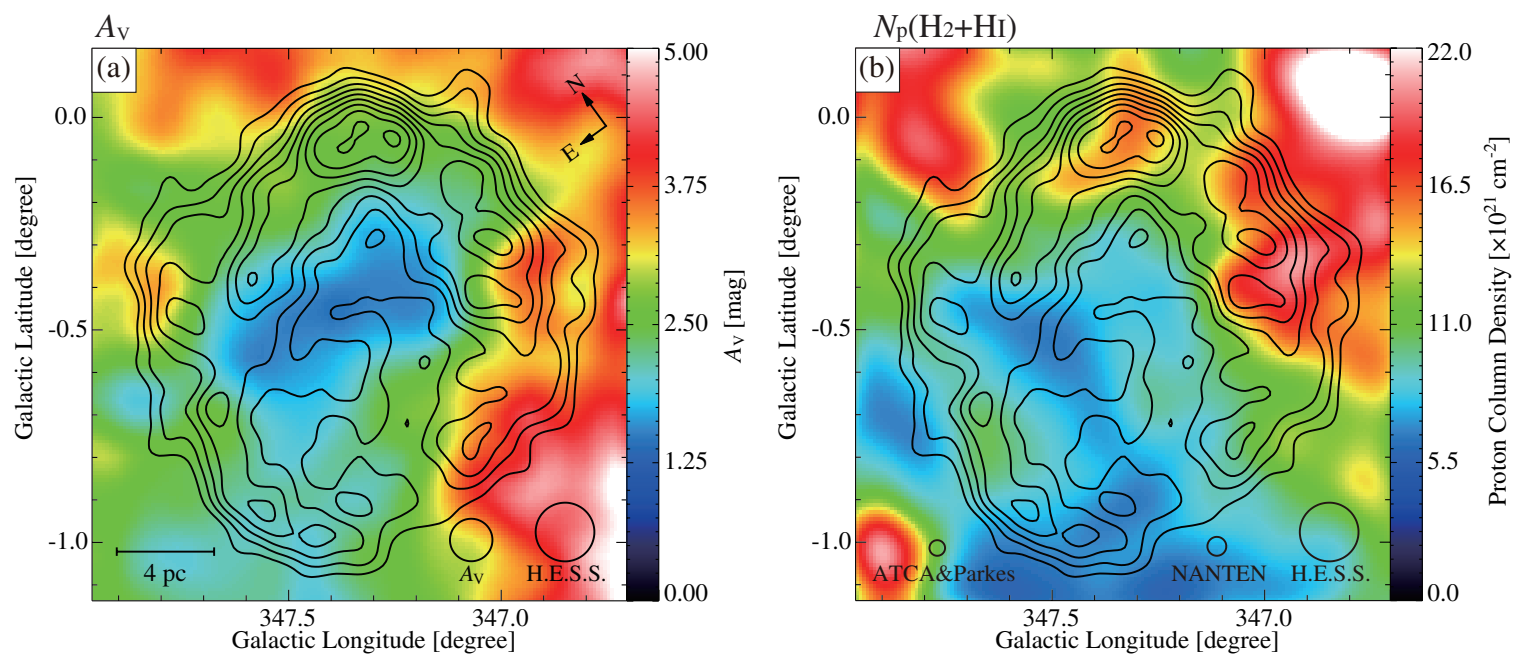

Figure 5. (a) $A_{\mathrm{V}}$ distribution (Dobashi et al. 2005) is shown in color. Contours are the same as in Figure 1(a). (b) Distribution of column density of the total ISM protons estimated from both $\mathrm{CO}$ and $\mathrm{H}$ in a velocity range from $V_{\mathrm{LSR}}=-20$ to $10 \mathrm{~km} \mathrm{~s}^{-1}$. Here, the Hi self-absorption is taken into account. Contours are the same as in Figure 1(a).

in the $\mathrm{CO} \mathrm{W}$ cloud. $\mathrm{H}$ I brightness $T_{L}(V)$ is expressed as follows (e.g., Sato \& Fukui 1978):

$$
\begin{aligned}
T_{L}(V)= & T_{\mathrm{s}}\left[1-e^{-\tau(V)}\right]+T_{L}^{\mathrm{FG}}(V)+\left[T_{L}^{\mathrm{BG}}(V)+T_{C}^{\mathrm{BG}}\right] e^{-\tau(V)} \\
& -\left(T_{C}^{\mathrm{FG}}+T_{C}^{\mathrm{BG}}\right),
\end{aligned}
$$

where $T_{L}(V), T_{\mathrm{s}}, \tau(V), T_{L}^{\mathrm{FG}}(V)$, and $T_{L}^{\mathrm{BG}}(V)$ are the observed H I brightness temperature, the spin temperature, the optical depth of cold $\mathrm{HI}$ in the cloud, and the foreground and background $\mathrm{HI}$ brightness temperature, respectively, at velocity $V$. $T_{C}^{\mathrm{FG}}$ and $T_{C}^{\mathrm{BG}}$ are the continuum brightness temperature at $21 \mathrm{~cm}$ wavelength in the foreground and background of the cloud, respectively. The radio continuum emission is weak in RX J1713.7-3946 (Lazendic et al. 2004), and $T_{C}^{\mathrm{FG}}$ and $T_{C}^{\mathrm{BG}}$ are nearly zero compared with $T_{L}(V)$.

We are then able to estimate the H I column density of dark H I clouds. Figure 4(b) shows the H I self-absorption dip with the background $\mathrm{H}$ I emission interpolated by a straight line connecting the two shoulders at $0 \mathrm{~km} \mathrm{~s}^{-1}$ and $20 \mathrm{~km} \mathrm{~s}^{-1}$. This gives a conservative estimate because the actual background $\mathrm{H}$ I shape perhaps has a more intense peak at $-10 \mathrm{~km} \mathrm{~s}^{-1}$ as seen in the northern area of the SNR. The spin temperature $T_{\mathrm{S}}$ of the dark H I gas is an unknown parameter. We estimate $T_{\mathrm{s}}$ to be less than $\sim 55 \mathrm{~K}$ from the lowest $\mathrm{H} \mathrm{I}$ brightness at the bottom of the dip in Figure 4(b) and higher than $\sim 20 \mathrm{~K}$, where the temperature of the $\mathrm{CO}$ clouds is $\sim 10 \mathrm{~K}$ (Sano et al. 2010). We estimate the absorbing dark H I column density to be $N_{\mathrm{p}}(\mathrm{HI})=1.0 \times 10^{21} \mathrm{~cm}^{-2}$ (optical depth $=0.8), 1.8 \times 10^{21} \mathrm{~cm}^{-2}$ (optical depth $=1.1$ ), and $3.1 \times 10^{21} \mathrm{~cm}^{-2}$ (optical depth $=1.5$ ) for three assumed cases $T_{\mathrm{s}}=30,40$, and $50 \mathrm{~K}$, respectively, for the half-power line width $\Delta v=10 \mathrm{~km} \mathrm{~s}^{-1}$, where the $\mathrm{H}$ I optical depth $\bar{\tau}$ is estimated by Equation (2) and $N_{\mathrm{p}}(\mathrm{HI})$ by the following relationship:

$$
N_{\mathrm{p}}(\mathrm{H} \mathrm{I})\left(\mathrm{cm}^{-2}\right)=1.823 \times 10^{18} T_{s}(\mathrm{~K}) \Delta v\left(\mathrm{~km} \mathrm{~s}^{-1}\right) \bar{\tau} .
$$

We shall here adopt $T_{\mathrm{S}}=40 \mathrm{~K}$ and a corresponding dark $\mathrm{H} \mathrm{I}$ optical depth of 1.1. A higher $T_{\mathrm{s}}$ gives a higher optical depth and vice versa. The relatively large optical depth around 1 is consistent with the fairly flat HI dip in Figure 4(b), which suggests weak saturation. We also tested the effects of elevating the background $\mathrm{HI}$ by $15 \mathrm{~K}$ and found a small change of $5 \times$
$10^{20} \mathrm{~cm}^{-2}$. The error is mainly introduced by the straight-line approximation and uncertainty in $T_{\mathrm{s}}$ of $\sim 10 \mathrm{~K}$. We infer that the dark H I column density is accurate within a systematic error of $\sim 1 \times 10^{21} \mathrm{~cm}^{-2}$.

The average $\mathrm{HI}$ density in the SE cloud is roughly estimated to be $150 \mathrm{~cm}^{-3}$ by dividing $1.8 \times 10^{21} \mathrm{~cm}^{-2}$ by $\sim 4 \mathrm{pc}$, the line-of-sight length of the thick ISM shell, following the three-dimensional model described in Section 3.5. This density is significantly lower than the critical density for collisional excitation of the CO $(J=1-0)$ transition, $\sim 1000 \mathrm{~cm}^{-3}$, consistent with no CO emission from the SE cloud and with a low spin temperature around $40 \mathrm{~K}$.

We also extended such an analysis to the regions with narrow $\mathrm{HI}$ dips associated with $\mathrm{CO}$ emission, where we adopt $T_{\mathrm{s}}=$ $10 \mathrm{~K}$, the kinetic temperature of the $\mathrm{CO}$ gas. The small dips in these regions indicate that the $\mathrm{H}$ i optical depth is generally as low as $\sim 0.1$, reflecting a small fraction of the residual $\mathrm{H}_{\mathrm{I}}$ in $\mathrm{CO}$ gas. We show the distributions of the peak optical depth of the H I self-absorption in Figure 6(a), and the derived total $\mathrm{HI}$ column density distribution, the sum of the $\mathrm{H}$ i in emission, and self-absorption in Figure 6(b), where the SE cloud is significant. We shall hereafter refer to the dark $\mathrm{H}$ I of $T_{\mathrm{S}}=40 \mathrm{~K}$ as "cool $\mathrm{HI}$ " and that of $T_{\mathrm{s}}=10 \mathrm{~K}$ as "cold H I."

\subsection{Total ISM Protons}

The number of the total ISM protons in the SNR is given by summing up the three components in a velocity range from -20 to $0 \mathrm{~km} \mathrm{~s}^{-1} ; \mathrm{H}_{2}$ derived from ${ }^{12} \mathrm{CO}(J=1-0)$, dark $\mathrm{H}_{\mathrm{I}}$ (dips) and warm $\mathrm{H}_{\mathrm{I}}$ (emissions). The results are shown as spatial distributions in Figure 7. Figures 7(a)-(d) show $N_{\mathrm{p}}\left(\mathrm{H}_{2}\right), N_{\mathrm{p}}(\mathrm{H} \mathrm{I})$, $N_{\mathrm{p}}\left(\mathrm{H}_{2}+\mathrm{H} \mathrm{I}\right)$, and $\mathrm{TeV} \gamma$-rays, respectively. We see that the total ISM protons $N_{\mathrm{p}}\left(\mathrm{H}_{2}+\mathrm{H}\right.$ I $)$ show a shell-like shape similar to the $\mathrm{TeV} \gamma$-rays, which significantly improves the correlation with the $\gamma$-rays compared with the case of molecular gas only. We therefore conclude that the contribution of $\mathrm{HI}$ as well as $\mathrm{H}_{2}$ is critical in counting the ISM protons. We find that in the south the total ISM proton is dominated by the atomic gas, whereas in the north the molecular and atomic protons are both important. A more quantitative comparison will be given in Section 3.5.2. Similar diagrams to Figure 7 of the total ISM protons are presented for the optically thin case for reference in Figure 15 

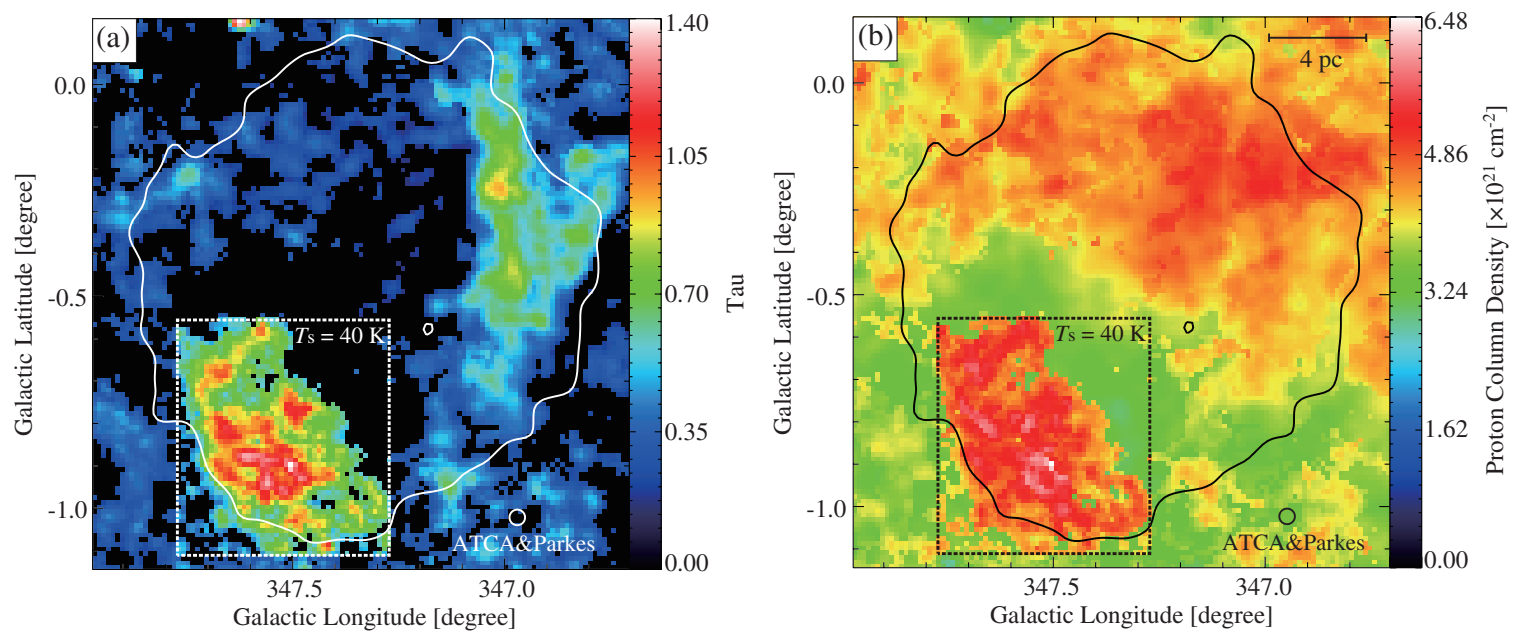

Figure 6. (a) Distribution of peak optical depth of the Hi self-absorption. (b) Distribution of atomic proton column density, $N_{\mathrm{p}}(\mathrm{HI})$, estimated for the H I emission and self-absorption. The velocity range in both the figures is from -20 to $0 \mathrm{~km} \mathrm{~s}^{-1}$. Contours show the H.E.S.S. TeV $\gamma$-ray distribution (Aharonian et al. 2007) and are plotted at 20 smoothed counts. We assume spin temperatures $T_{\mathrm{s}}$ of $40 \mathrm{~K}$ and $10 \mathrm{~K}$, inside and outside the dotted box toward the SE cloud, respectively.
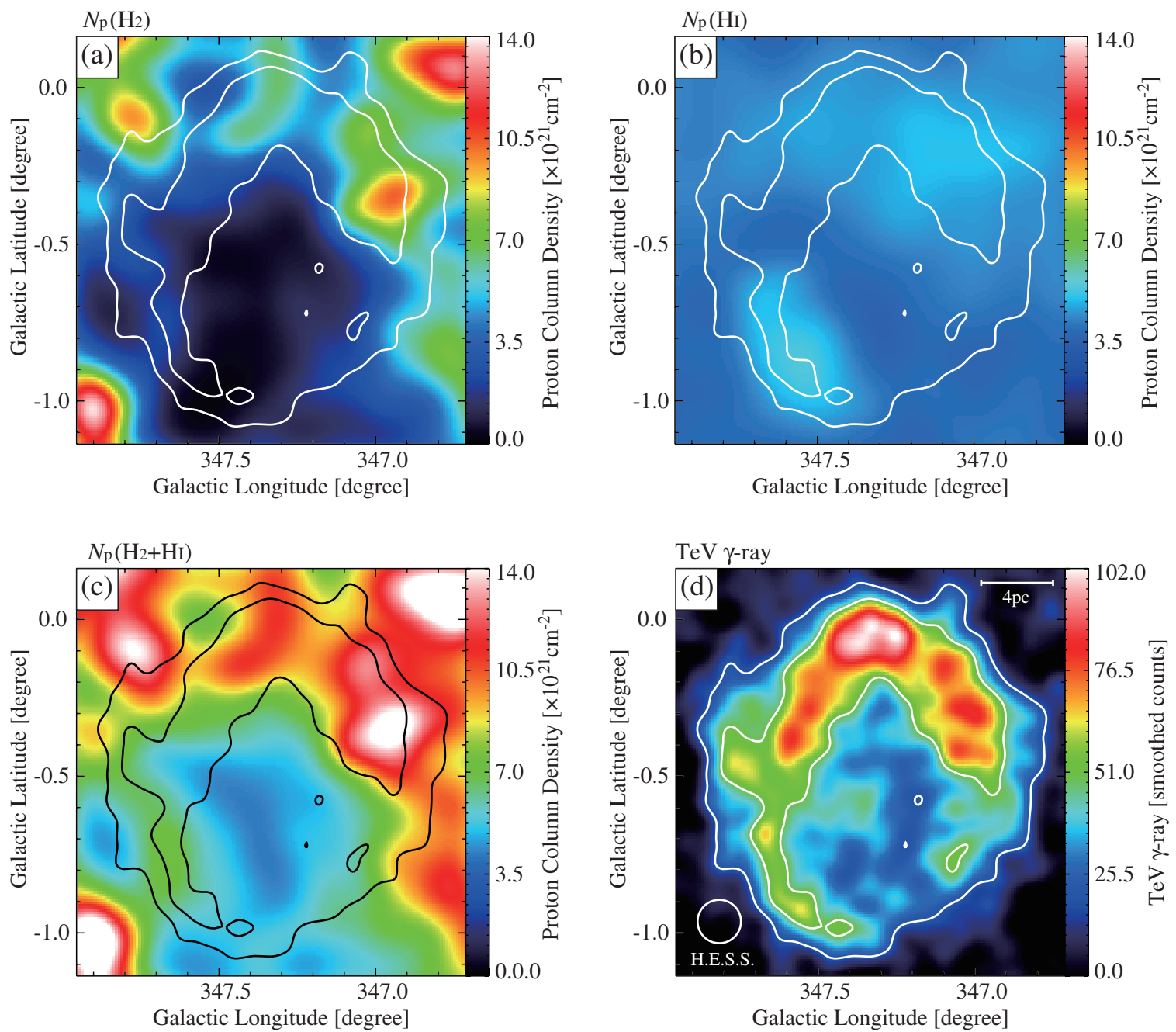

Figure 7. (a-c) Distributions of ISM proton column density, $N_{\mathrm{p}}$, are shown. All the data sets used for the three images are smoothed to have an HPBW of 8.3 , the same figure as the HPBW of H.E.S.S. (a) $N_{\mathrm{p}}\left(\mathrm{H}_{2}\right)$ estimated from ${ }^{12} \mathrm{CO}(J=1-0)$. (b) $N_{\mathrm{p}}(\mathrm{HI})$ estimated from H I with correction of self-absorption. (c) $N_{\mathrm{p}}\left(\mathrm{H}_{2}+\mathrm{H} \mathrm{I}\right)$ estimated by summing up of $N_{\mathrm{p}}\left(\mathrm{H}_{2}\right)$ and $N_{\mathrm{p}}(\mathrm{HI})$. (d) TeV $\gamma$-ray distribution. Contours are plotted every 50 smoothed counts from 20 smoothed counts. 

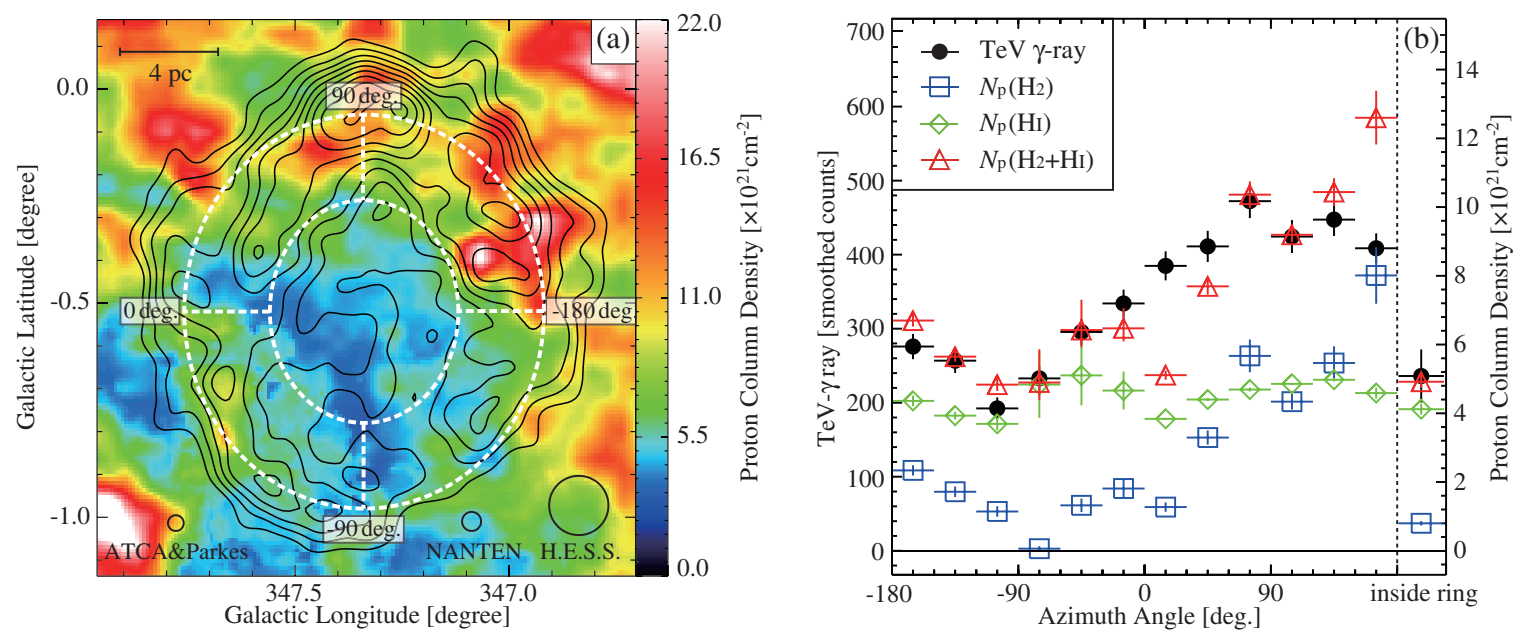

Figure 8. (a) Distributions of column density of the total ISM protons $N_{\mathrm{p}}\left(\mathrm{H}_{2}+\mathrm{HI}\right)$ in a velocity range from -20 to $0 \mathrm{~km} \mathrm{~s}^{-1}$. Contours are the same as in Figure 1 (a). (b) Azimuthal distributions of $N_{\mathrm{p}}\left(\mathrm{H}_{2}\right), N_{\mathrm{p}}(\mathrm{H} \mathrm{I}), N_{\mathrm{p}}\left(\mathrm{H}_{2}+\mathrm{H} \mathrm{I}\right)$, and $\mathrm{TeV} \gamma$-ray-smoothed counts per beam between the two elliptical rings shown in (a). The proton column densities are averaged values between the rings (see the text). Semimajor and semiminor radii of the outer ring are 0.46 and 0.42 , respectively, and the radii of the inner ring are half of them. The same plots inside the inner ring are shown on the right side of (b).

in Appendix C, where the shell-like distribution toward the SE cloud is missing.

\subsection{The $\gamma$-Rays and the ISM Protons \\ 3.5.1. Gamma-ray Distribution}

The $\mathrm{TeV} \gamma$-ray distribution obtained by H.E.S.S. is a nearly circular-symmetric shell with some ellipticity elongated in the north-south direction. In order to gain an insight into the distribution of the $\gamma$-ray emissivity we undertake a simple analysis of the $\gamma$-ray distribution. We first adopt an elliptical annular ring in the analysis, while Aharonian et al. (2006b) made a similar analysis by using a circular annular ring in correlating $\gamma$-rays and NANTEN CO intensity (see Figure 17 in Aharonian et al. 2006b).

We estimated the radius of the $\gamma$-ray shell as defined at a half-intensity level of the peak $\gamma$-ray smoothed counts every $15^{\circ}$ for an assumed center. We averaged the radii in angle and minimized the sum of the squares of the deviation from the average. This process gives a central position to be $(l, b)=(347.34,-0.52)$. For this central position, we plotted the radius every $15^{\circ}$ and found that a sinusoidal distribution is a reasonable approximation as expected. Fitting this plot by a sinusoidal curve, we find that the shell is approximated by an elliptical shape with an aspect ratio of 1.1 whose major axis is almost in the north-south direction. This elliptical shape is adopted in Figure 8(a).

Figure 9 shows the radial scatter of $\gamma$-ray smoothed counts and an averaged value shown by a step function in radius $r$ every 0.05 . Here, we also adopted the elliptical shape and normalized the radius to that of the major axis with the elliptical modification. After several trials of different functional forms, we found that a Gaussian radial distribution of the $\gamma$-ray emissivity per volume reproduces the projected radial distribution well in Figure 9. In the fitting we have two free parameters of the Gaussian shape, the peak radius $r_{0}$ and the sigma $\sigma$, expressed as follows:

$$
F(r)=A \times e^{-\left(r-r_{0}\right)^{2} / 2 \sigma^{2}},
$$

where $A$ is a normalization coefficient. By requiring that the error in the fitting becomes minimum in the projected distribution

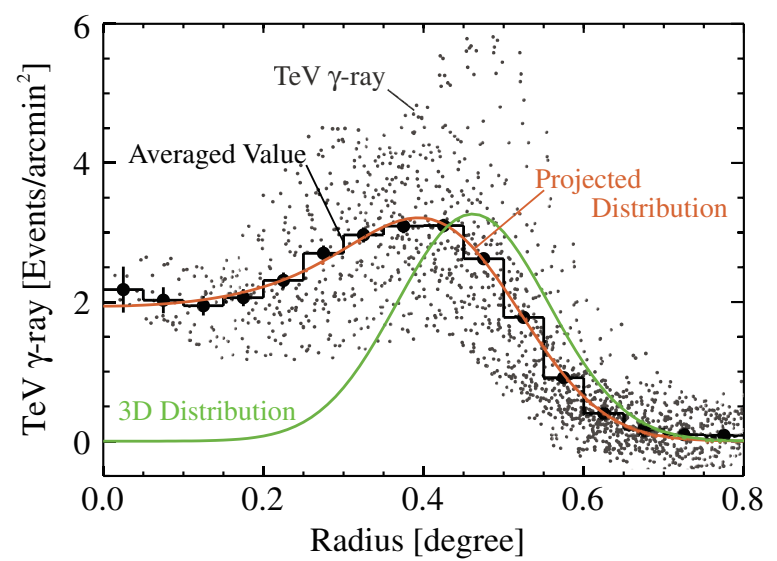

Figure 9. Radial distribution of $\mathrm{TeV} \gamma$-ray radiation. Small dots show the distributions of all the H.E.S.S. data points and large filled circles with error bars show averaged values at each radius. We assume a 3D spherical shell with a Gaussian-like intensity distribution along its radius to approximate the $\mathrm{TeV}$ $\gamma$-ray distribution (see the text). The green line shows the estimated 3D Gaussian distribution and the red line shows its projected distribution. The peak radius and the FWHM of the green line are estimated to be $0.46(\sim 8.0 \mathrm{pc})$ and 0.24 $(\sim 4.2 \mathrm{pc})$, respectively.

shown by the step function, we found that $r_{0}=0.46$ and $\sigma=$ 0.10 give the best fit as shown in Figure 9. This distribution shows that the observed shell is consistent with a shell of a halfintensity thickness $\sim 0.24$ with nearly zero emissivity toward the center. This analysis indicates that the $\gamma$-rays are mainly emitted in a thick shell of $8.0 \mathrm{pc}$ radius and $4.2 \mathrm{pc}$ width at the halfintensity level with nearly zero emission from the inner part. A similar thick-shell model was also obtained by Aharonian et al. (2006a). Numerical modeling of the $\gamma$-ray emission has been undertaken by several authors and indicates that the $\gamma$-ray emission has a rather steep gradient beyond the peak of the shell in either of the leptonic or hadronic scenario (e.g., Jun \& Norman 1996; Zirakashvili \& Aharonian 2010). The fitting to the H.E.S.S. data above shows that the gradient in the $\gamma$-ray distribution is not so steep toward the outside, which may be due to smearing in space by averaging. We shall not try a further elaborated analysis here due to the limiting angular resolution of H.E.S.S., which is 0.14 (FWHM). 
Figure 9 shows that the projected radial distribution of ISM protons follows a fairly similar distribution to the $\gamma$-rays inside the SNR. This is consistent with the ISM distribution also being shell-like with an inner cavity as is consistent with the stellarwind shell discussed in Section 4.1; if the ISM has no cavity in the inner part, the projected distribution of the ISM should increase toward the center. We shall assume hereafter that the ISM distribution is also approximated by the same Gaussian shape as the $\gamma$-rays with a radius of $8.0 \mathrm{pc}$ with a thickness of $4.2 \mathrm{pc}$ at the half-intensity level.

\subsubsection{Comparison between the $\gamma$-Rays and the ISM Protons}

In the hadronic scenario, the target distribution should be correlated with the $\gamma$-ray distribution for a uniform CR distribution. This correlation should be seen inside the shell of the SN shock which has a sharp gradient beyond its outer radius. We expect that the ISM protons are distributed beyond the outermost edge of the shell where $\mathrm{CR}$ protons cannot reach by diffusion. Beyond the SNR shock, the $\gamma$-ray emission profile may be influenced by components from the diffuse CR background and by the energydependent transport of escaping CRs from RX J1713.7-3946 into the clumpy ISM (e.g., Gabici et al. 2009; Casanova et al. 2010a, 2010b). We are able to avoid possible effects of such cutoff by taking the radius of the correlation analysis well within the SNR shell where the CR protons do not decrease in energy density.

The ISM proton distribution is shown in Figure 8(a) with the two annular elliptical rings along the shell, where the size of the outer ring was chosen to meet the requirement above. Figure 8(b) shows a comparison between the ISM protons and $\gamma$-rays in the position angle shown in Figure 8(a), where the vertical scale is adjusted so that the correspondence with the $\mathrm{TeV} \gamma$-rays becomes optimum. Here, the error in the $\mathrm{TeV} \gamma$-ray emission from the publicly available H.E.S.S. image is approximately (smoothed counts) ${ }^{0.5}$. In Figure $8(\mathrm{~b})$, the uncertainty in the dark $\mathrm{HI}_{\mathrm{I}}$ in the SE cloud, $1 \times 10^{21} \mathrm{~cm}^{-2}$, is on the order of $10 \%-20 \%$ of the total. The total ISM proton density shows good agreement with the $\mathrm{TeV} \gamma$-ray angular distribution and also the central part in the inner ring. We recall that $\mathrm{CO}$ alone showed marked deficiency toward the SE cloud compared with the $\gamma$-rays (see Figure 17 of Aharonian et al. 2006b). The present analysis indicates that the deficiency is recovered by including $\mathrm{HI}$ and shows that the total gas of both atomic and molecular components has a good correlation with the $\mathrm{TeV} \gamma$-rays in the annular ring. The total mass of the ISM protons responsible for the $\gamma$-rays is $2.0 \times 10^{4} M_{\odot}$ over the whole SNR (radius $0.65)$; the mass of molecular protons is $0.9 \times 10^{4} M_{\odot}$ and that of atomic protons is $1.1 \times 10^{4} M_{\odot}$, where we assume that the ISM protons interacting with the CR protons is proportional to the TeV $\gamma$-rays (Section 3.5.1., Figure 10).

There are two points in Figure 8(b) for which additional remarks may be appropriate. One is the point at an azimuth angle of $115^{\circ}$ which may be estimated too low due to the lack of correction for the self-absorption because of the large velocity shift in the expanding shell (see Figure 12 in Appendix B). Another is the point at an azimuth angle of $165^{\circ}$ where the strong CO emission (peak A after Fukui et al. 2003) increases the proton column density, although the increased protons may not be interacting with the CR protons beyond the SNR shock, leading to less $\gamma$-rays.

An independent test is made by the radial distribution of the ISM protons given in Figure 10, where an average taken over the same binning as the $\gamma$-rays in Figure 9 is shown by a step

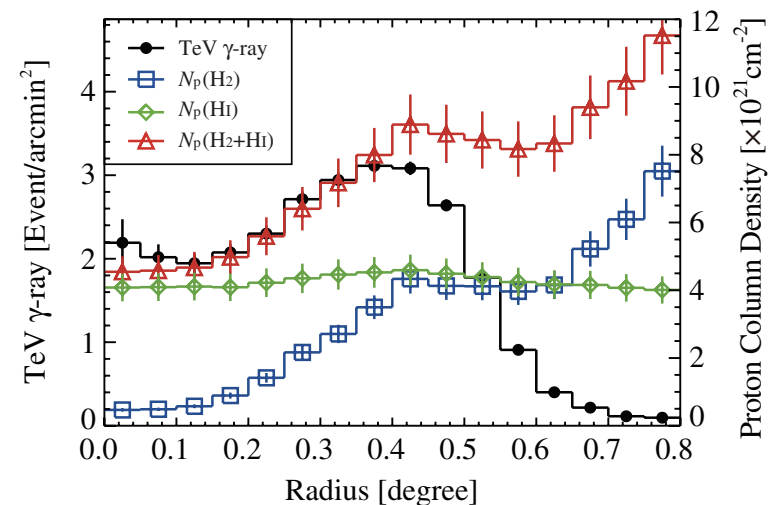

Figure 10. Radial distributions of averaged values of $\mathrm{TeV} \gamma$-ray radiation, $N_{\mathrm{p}}\left(\mathrm{H}_{2}\right), N_{\mathrm{p}}(\mathrm{HI})$, and $N_{\mathrm{p}}\left(\mathrm{H}_{2}+\mathrm{H} \mathrm{I}\right) . N_{\mathrm{p}}\left(\mathrm{H}_{2}\right)$ and $N_{\mathrm{p}}(\mathrm{H} \mathrm{I})$ show column densities estimated from ${ }^{12} \mathrm{CO}(J=1-0)$ and $\mathrm{H}$, respectively, and $N_{\mathrm{p}}\left(\mathrm{H}_{2}+\mathrm{H} \mathrm{I}\right)$ shows the total ISM proton column density.

function, and the total ISM protons and $\gamma$-rays are superposed with the same proportional factor as adopted in Figure 8. Here, the error in the $\mathrm{TeV} \gamma$-ray emission is approximately (oversampling-corrected total smoothed count $)^{0.5}$ normalized to $1(\operatorname{arcmin})^{2}$. We see that the $N_{\mathrm{p}}\left(\mathrm{H}_{2}+\mathrm{H}_{\mathrm{I}}\right)$ and $\gamma$-rays show a good agreement inside the shell and the $\gamma$-rays sharply decrease outside the shell. This offers another presentation of the good correlation between the $\gamma$-rays and the ISM protons.

We argue that the apparent anti-correlation between the $\mathrm{HI}_{\mathrm{I}}$ brightness at the bottom of the dips and the $\gamma$-rays in the SE cloud (Figure 4) is consistent with the H I dips being due to the cool and dense $\mathrm{H}$ I gas. The anti-correlation is interpreted that the spin temperature $T_{\mathrm{S}}$ of H I decreases with density (Section 3.3) and that the $\gamma$-rays increase with the ISM proton density locally in the SE cloud, demonstrating detailed correspondence between the $\gamma$-rays and the ISM protons, which is mainly atomic. The small and narrow $\mathrm{HI}$ dips in the $\mathrm{W}$ and $\mathrm{N}$ clouds have the $\mathrm{HI}$ column density less than $10^{20} \mathrm{~cm}^{-2}$, significantly lower than the typical molecular column density by two orders of magnitude. So, in most of the regions except for the SE cloud the H I column density is dominated by emission but not by self-absorption. For the sake of reference, we show a set of similar diagrams of ISM proton distributions for the optically thin case in Figures 16 and 17 in Appendix C, corresponding to Figures 8 and 10, respectively.

Before concluding this subsection, we cautiously note that the cool/cold Hi could not be estimated accurately, if the cool/cold $\mathrm{H}_{\mathrm{I}}$ is optically thick, if the cool/cold $\mathrm{H}$ I lies behind optically thick foreground $\mathrm{H}_{\mathrm{I}}$ in the line of sight, or if the background $\mathrm{H}$ I profile has a different shape from its neighbors. Such effects, while posing intrinsic limits for probing cool/cold $\mathrm{HI}$, are relatively unimportant for nearby objects at a distance of $1 \mathrm{kpc}$ or less where foreground $\mathrm{H}$ i is not important. The dark $\mathrm{HI}_{\mathrm{I}} \mathrm{W}$ and SE clouds are probably good examples where the cool/cold $\mathrm{H}_{\mathrm{I}}$ is well traced by the low $\mathrm{H}_{\mathrm{I}}$ brightness, whereas the $\mathrm{N}$ cloud with higher $\mathrm{H}$ I brightness may be partially affected by the foreground $\mathrm{H}_{\mathrm{I}}$ in the line of sight.

\section{DISCUSSION}

\subsection{The Evacuated Cavity by the Stellar Wind}

It is likely that the CO shell in Figure 1(b) was formed over a timescale of Myr by the stellar wind of the progenitor, an $\mathrm{OB}$ 
Table 1

A Comparison between RX J1713.7-3946 and Pegasus Loop

\begin{tabular}{lcc}
\hline \hline & RX J1713.7-3946 & Pegasus Loop $^{\mathrm{b}}$ \\
\hline Distance $(\mathrm{kpc})$ & 1 & 0.1 \\
Diameter (pc) & 17.4 & 25 \\
Total mass of the ISM $\left(M_{\odot}\right)$ & $\sim 20000^{\mathrm{c}}$ & $\sim 1500$ \\
Thickness of the ISM shell $(\mathrm{pc})$ & $\sim 4.2$ & $\sim 5$ \\
Peak brightness of H I $(\mathrm{K})$ & $\sim 170$ & $\sim 40$ \\
Line width of $\mathrm{H}\left(\mathrm{km} \mathrm{s}^{-1}\right)$ & $\sim 20$ & $\sim 16$ \\
Expansion velocity of the gaseous shell $\left(\mathrm{km} \mathrm{s}^{-1}\right)$ & $\sim 10$ & $\sim 7-9$ \\
Spectral type of the progenitor & B1 V/B0 V & B2 IV \\
\hline
\end{tabular}

Notes. The Pegasus loop may consist of two shells and the mass should be regarded as an upper limit (Yamamoto et al. 2006).

${ }^{\text {a }}$ Fukui et al. (2003); Moriguchi et al. (2005)

b Yamamoto et al. (2006)

c Present work

${ }^{\mathrm{d}}$ Cassam-Chenaï et al. (2004)

star that exploded as a supernova (SN) $1600 \mathrm{yr}$ ago. The total velocity span of the CO shell, $\sim 20 \mathrm{~km} \mathrm{~s}^{-1}$, is much smaller than the SN shock speed and indicates that it takes Myr to form the shell of the ISM as roughly estimated by dividing the radius $9 \mathrm{pc}$ by $10 \mathrm{~km} \mathrm{~s}^{-1}$. Molecular gas expanding at $10 \mathrm{~km} \mathrm{~s}^{-1}$ can move only $0.01 \mathrm{pc}$ in $1000 \mathrm{yr}$. Therefore, the current $\mathrm{CO}$ distribution has little been affected by the SN explosion and holds the initial condition before the shock interaction.

While a stellar-wind shell with a known central star is not often observed elsewhere, one such example is the Pegasus loop found in ${ }^{12} \mathrm{CO}(J=1-0), \mathrm{HI}$, and dust emission at $(l, b)=\left(109^{\circ},-45^{\circ}\right)$ centered on a runway star HD886 (B2 IV) (Yamamoto et al. 2006). The Pegasus loop is located at $\sim 100 \mathrm{pc}$ in a relatively uncontaminated environment outside the Galactic plane. No SN explosion has occurred yet in this shell. A comparison between RX J1713.7-3946 and the Pegasus loop is given in Table 1. In Pegasus the swept-up shell of the ISM has a width of $\sim 5 \mathrm{pc}$ for a radius of $\sim 18 \mathrm{pc}$ and a total mass of $\sim 1500 M_{\odot}$. The shell is mostly atomic and consists of 78 smaller ${ }^{12} \mathrm{CO}(J=1-0)$ clumps (see Figure 10 in Yamamoto et al. 2006). The clumped CO is a natural outcome of thermal/ gravitational instability and seems common in such a shell. The shell is expanding at a total velocity span of $15 \mathrm{~km} \mathrm{~s}^{-1}$. The H I density inside the shell is $\sim 1 \mathrm{~cm}^{-3}$ in the north, where the stellar wind evacuated the ISM over 1 Myr. The Pegasus loop is located in a somewhat lower-density environment than RX J1713.7-3946 and offers an insight into the initial condition of the ISM prior to the SN explosion in RX J1713.7-3946.

Inoue et al. (2009) and IYIF2012 carried out numerical simulations of the hydrodynamical interaction between the shock wave and the highly inhomogeneous neutral gas to model the interaction in RX J1713.7-3946. The SN in RX J1713.7-3946 exploded in the cavity with average density less than $1 \mathrm{~cm}^{-3}$ (e.g., Zirakashvili \& Aharonian 2010; Morlino et al. 2009; Berezhko \& Völk 2008) and the dense shell with $\mathrm{CO}$ clumps remaining more or less as they were prior to the $\mathrm{SN}$ explosion. The SN shock front moves almost freely at $\geqslant 3000 \mathrm{~km} \mathrm{~s}^{-1}$ in the cavity in the early phase of $\sim 1000 \mathrm{yr}$ and begins to interact with the dense and thick clumpy ISM wall swept up by the stellar wind only in the last few $100 \mathrm{yr}$. The $\gamma$-ray shell is not strongly deformed, while we see some deviations of a parsec-scale from a perfect circular shell, suggesting effects of recent dynamical interaction.

The interaction between molecular clumps and the shock is observed as the X-ray enhancement around dense molec- ular clumps at a spatial resolution higher than 0.5 pc. Sano et al. (2010) showed that the molecular clump peak $\mathrm{C}$ is rimbrightened in X-rays, suggesting that it is a dense clump overtaken by the shock, and peak A (Fukui et al. 2003) is also X-ray brightened only toward its inner edge, indicating the shock interaction at the inner boundary of peak A. IYIF2012 showed that the initial magnetic field $B$ of $1 \mu \mathrm{G}$ is amplified to $0.1-1 \mathrm{mG}$ near dense clumps by the enhanced turbulence driven by the shock. The stronger magnetic field explains the X-ray enhancement as being due to the enhanced synchrotron emission that is proportional to $B^{2}$, or due to increased acceleration. IYIF2012 also showed that the shock speed $v_{s}$ is significantly reduced locally with density $n\left(\mathrm{~cm}^{-3}\right)$ such that $v_{s} \sim 3000 \mathrm{~km} \mathrm{~s} / \sqrt{n / n_{0}}$, where $n_{0}=1 \mathrm{~cm}^{-3}$. This dependence of $v_{s}$ on density can explain the absence of thermal X-rays in the SNR because the molecular gas is too dense to be affected by the shock to emit thermal X-rays (IYIF2012). A uniform lower-density case with significant thermal X-rays by shock heating is presented by Ellison et al. (2010) but such a model is not applicable to the highly inhomogeneous ISM of RX J1713.7-3946 (IYIF2012; see also the discussion in Section 4 of Ellison et al. 2010). The picture above is also consistent with peak $\mathrm{C}$ having density greater than $10^{4} \mathrm{~cm}^{-3}$ and has survived without erosion (Sano et al. 2010).

\subsection{The $\gamma$-Ray Emission Mechanism}

$\mathrm{TeV} \gamma$-rays are emitted via two mechanisms, either leptonic or hadronic processes. The leptonic process explains $\gamma$-rays via the inverse Compton effect between CR electrons and lowenergy photons. In the hadronic scenario, $\gamma$-rays are emitted by the decay of neutral pions which are produced in the high-energy reactions between CR protons and ISM protons. DSA is the most widely accepted scheme of particle acceleration (Bell 1978; Blandford \& Ostriker 1978; Jones \& Ellison 1991; Malkov \& O'C Drury 2001). The previous works on RX J1713.7-3946 show that the observed spectral energy distribution of $\gamma$-rays and $\mathrm{X}$-rays is explained by the leptonic and/or hadronic mechanisms if DSA works to accelerate the particles (Aharonian et al. 2006b; Porter et al. 2006; Katz \& Waxman 2008; Berezhko \& Völk 2008; Ellison \& Vladimirov 2008; Tanaka et al. 2008; Morlino et al. 2009; Acero et al. 2009; Ellison et al. 2010; Patnaude et al. 2010; Zirakashvili \& Aharonian 2010; Abdo et al. 2011; Fang et al. 2011). 
In the hadronic scenario, where the neutral pion decay determines the $\gamma$-rays via proton-proton reactions, the average density of the target protons is constrained by the total energy of CR protons; an average target density greater than $0.1 \mathrm{~cm}^{-3}$ is required to produce $\mathrm{CR}$ protons having the total energy of $10^{51} \mathrm{erg}$, for the maximum energy of the SN explosion, while higher target density is required for less CR proton energy. In the leptonic scenario, where the inverse Compton process produces $\gamma$-rays, the critical parameter is the magnetic field which constrains the synchrotron loss timescale of CR electrons; a magnetic field of the order of $10 \mu \mathrm{G}$ is usually required (e.g., Tanaka et al. 2008).

We argue here that the highly inhomogeneous distribution of the ISM, the cavity, and the dense and clumpy wall opens up the possibility of accommodating the low-density site for DSA and the high-density target simultaneously as discussed into detail by IYIF2012. A similar argument on the hadronic interaction between $\mathrm{CR}$ protons with the ambient dense clouds has been presented by Zirakashvili \& Aharonian (2010). In this picture, first, the CRs are accelerated via DSA in the lowdensity cavity, and second, the $\mathrm{CR}$ protons reach and react with the target protons in the dense wall to produce $\gamma$-rays. The main energy range of the $\mathrm{CR}$ protons required for hadronic $\mathrm{TeV} \gamma$-rays is $10-800 \mathrm{TeV}$ (Zirakashvili \& Aharonian 2010). The penetration depth, $l_{\mathrm{pd}}$, of the CRs is expressed as follows (IYIF2012):

$$
\begin{aligned}
l_{\mathrm{pd}} \sim & 0.1 \eta^{1 / 2}\left(\frac{E}{10 \mathrm{TeV}}\right)^{1 / 2}\left(\frac{B}{100 \mu \mathrm{G}}\right)^{-1 / 2} \\
& \times\left(\frac{t_{\mathrm{age}}}{10^{3} \mathrm{yr}}\right)^{1 / 2}(\mathrm{pc}),
\end{aligned}
$$

where $E, B$, and $t_{\text {age }}$ are the particle energy, the magnetic field, and the age of the SNR. The parameter $\eta$ is the socalled gyro-factor and has some ambiguity. In the SNR, it is reasonable to consider $\eta \sim 1$ at least around the cloud (Uchiyama et al. 2007). Thus, the penetration depth of the protons in the above energy range is $0.3-2.8 \mathrm{pc}$ for the magnetic field of $10 \mu \mathrm{G}$ and $0.1-0.9 \mathrm{pc}$ for $100 \mu \mathrm{G}$ in a typical timescale of $\sim 10^{3}$ yr. The penetration depth of the CR electrons is determined by taking $t_{\text {age }}$ equal to the synchrotron loss timescale (e.g., Tanaka et al. 2008) in Equation (6) and becomes energy independent for the X-ray emitting electrons of $1-40 \mathrm{TeV}$ as follows:

$$
l=0.026 \eta^{1 / 2}\left(\frac{B}{100 \mu \mathrm{G}}\right)^{-3 / 2}(\mathrm{pc}) .
$$

We estimate $l$ to be from $0.8 \mathrm{pc}$ for $10 \mu \mathrm{G}$ to $0.026 \mathrm{pc}$ for $100 \mu \mathrm{G}$ if $\eta=1$. CR protons can therefore reach and penetrate into the dense gas within the parsec-scale of the acceleration site to produce $\mathrm{TeV} \gamma$-rays, while the CR electrons stay relatively closer to the acceleration site, in particular, near the dense gas having a strong magnetic field. This offers an explanation for the hadronic $\gamma$-ray production, and the correlation between the $\gamma$-rays and target protons in Figures 4, 8, and 10 is a natural outcome in the scenario (IYIF2012).

Gabici et al. (2007) discussed the importance of the energydependent interaction between $\mathrm{CR}$ protons and molecular clouds, and Zirakashvili \& Aharonian (2010) discussed that the $\gamma$-ray spectrum may not distinguish the leptonic and hadronic scenarios in the case of RX J1713.7-3946 due to such energy dependence. Recently, Fermi Large Area Telescope (LAT) ob- servations showed that the GeV spectrum of RX J1713.7-3946 is hard, similar to what is expected in the leptonic scenario, and Abdo et al. (2011) discussed that the hard spectrum may favor the leptonic scenario. IYIF2012, however, argued that the hard Fermi-LAT GeV spectrum is also explained well by the hadronic scenario being due to the energy-dependent penetration of $\mathrm{CR}$ protons into the dense clouds and that the leptonic scenario explaining the spectrum is not unique. IYIF2012 confirmed that the $\gamma$-ray spectrum becomes similar for both the leptonic and hadronic scenarios, and is not usable to distinguish the two scenarios, as noted by Zirakashvili \& Aharonian (2010), and concluded that the hadronic origin is testable only by comparing $\gamma$-rays with the ISM target distribution. The present results have demonstrated that the ISM proton distribution indeed shows a good spatial correspondence with the $\gamma$-rays by taking into account the contribution of the $\mathrm{HI}$, and matches the prediction by Zirakashvili \& Aharonian (2010) and IYIF2012.

The total energy of CR protons is estimated by the relationship between the total target protons and the observed $\gamma$-rays (2-400 TeV) after extrapolating the proton spectrum to $1 \mathrm{GeV}$ as follows (Aharonian et al. 2006b):

$$
W_{\mathrm{tot}} \sim(1-3) \times 10^{50}\left(\frac{d}{1 \mathrm{kpc}}\right)^{2}\left(\frac{n}{1 \mathrm{~cm}^{-3}}\right)^{-1}(\mathrm{erg})
$$

where the distance to the source is $d \sim 1 \mathrm{kpc}$ and the density of the target protons is $n$. The average density of ISM protons is calculated to be $\sim 130 \mathrm{~cm}^{-3}$ for the total mass of the ISM protons $2.0 \times 10^{4} M_{\odot}$ over the whole SNR (radius 0.65 ) as modeled in Figure 10 and the total CR proton energy is calculated to be $\sim(0.8-2.3) \times 10^{48}$ erg by using Equation (7). This corresponds to $\sim 0.1 \%$ of the total energy release of the SN explosion and may appear low. The other SNRs like W44 and W28 of a few to 10 times $1000 \mathrm{yr}$ old have a total CR proton energy on the order of $10^{49}-10^{50}$ erg (Abdo et al. 2010; Giuliani et al. 2010). We may speculate that the CR protons become accumulated in a few 10,000 yr to reach more than $10 \%$ of the SN explosion energy. This issue is to be further tested by examining CR escaping from SNRs (e.g., Gabici et al. 2009; Casanova et al. 2010a, 2010b).

To summarize the discussion, we have shown that a combined analysis of $\mathrm{CO}$ and $\mathrm{HI}$ provides a reasonable candidate for the target ISM protons and thereby lends new support to the hadronic scenario. We should note that the present analysis offers one of the necessary conditions for the hadronic scenario for uniform CR proton distribution, but it is not a full verification of the hadronic scenario and does not rule out leptonic components. We need to acquire additional observations before fully establishing the hadronic scenario, including a better determination of the magnetic field and higher angular resolution images of $\gamma$-rays at least comparable to that of the ISM. The Cherenkov Telescope Array will provide such images in the future. We discussed that the observed highly inhomogeneous distribution of the ISM plays an essential role in the $\gamma$-ray production; DSA works in a highly evacuated cavity and the accelerated CR protons travel over a parsec to interact with the surrounding dense ISM protons. It is important to develop a similar analysis of both $\mathrm{HI}$ and CO in the other similar objects like RX J0852.0-4622 (Vela Jr.), RCW86, and HESS J1731-347. Such works are in progress based on the NANTEN2 observations and highresolution $\mathrm{H} \mathrm{I}$ interferometry. 


\section{CONCLUSIONS}

We summarize the main conclusions as follows.

1. A new analysis of $\mathrm{CO}$ and $\mathrm{HI}$ has revealed that the $\mathrm{TeV}$ $\gamma$-ray SNR RX J1713.7-3946 is associated with a significant amount of $\mathrm{H}_{\mathrm{I}}$ gas without $\mathrm{H}_{2}$ derived from CO. This H I gas is relatively dense and cold and detectable mainly as $\mathrm{Hi}$ emission. We have also identified regions where $\mathrm{HI}_{\mathrm{I}}$ is observed as dark $\mathrm{HI}_{\mathrm{I}}$ in selfabsorption dips and derived the total ISM proton column density over the SNR. The $\mathrm{H}_{\mathrm{I}}$ plus $\mathrm{H}_{2}$, the total number of ISM protons, provides one of the necessary conditions, target protons, in the hadronic origin of the $\gamma$-rays. Such target ISM protons have not been identified in the previous study that took into account only $\mathrm{H}_{2}$, although the present finding alone does not exclude the leptonic origin.

2. For an annular pattern around the $\mathrm{TeV} \gamma$-ray shell, we compared the total ISM proton distribution with the TeV $\gamma$-ray distribution and found that they show reasonably good correspondence, varying by similar factors. The inclusion of the atomic protons observed as the H I selfabsorption dips is essential particularly in the southeast of the $\gamma$-ray shell. The interpretation of $\mathrm{H}_{\mathrm{I}}$ self-absorption dips is also supported by the enhanced optical extinction toward the southeast rim.

3. The cavity surrounding the SNR was created by the stellar wind of the SN progenitor. The inside of the cavity is of low density with $<1 \mathrm{~cm}^{-3}$ while the cavity wall consists of the dense and clumpy atomic or molecular target protons of $\geqslant 100-1000 \mathrm{~cm}^{-3}$. The DSA in the highly inhomogeneous ISM offers a reasonable mechanism of particle acceleration in the low-density cavity, and the dense wall acts as the target for $\gamma$-ray production by the CR protons. Hydro-dynamical numerical simulations of the interaction have shown the detailed physical processes involved (IYIF2012).

4. By considering the other pieces of the observational and theoretical works accumulated thus far, the present results make the hadronic interpretation much more comfortable in RX J1713.7-3946. The current energy of the total CR protons is estimated to be $\sim 10^{48} \mathrm{erg}, 0.1 \%$ of the total energy of the $\mathrm{SN}$ explosion, if we assume that the $\gamma$-rays are all produced by the hadronic process.

We are grateful to Felix Aharonian for the lively and fruitful discussion on the subject, without which the work would not have been completed. The NANTEN project is based on a mutual agreement between Nagoya University and the Carnegie Institution of Washington (CIW). We greatly appreciate the hospitality of all the staff members of the Las Campanas Observatory of CIW. We are thankful to many Japanese public donors and companies who contributed to the realization of the project. NANTEN2 is an international collaboration of 10 universities: Nagoya University, Osaka Prefecture University, University of Cologne, University of Bonn, Seoul National University, University of Chile, University of New South Wales, Macquarie University, University of Sydney, and University of ETH Zurich. This work is financially supported by a grantin-aid for Scientific Research (KAKENHI, No. 15071203, No. 21253003, No. 20244014, No. 23403001, No. 22540250, No. 22244014, No. 23740149-01, and No. 22740119) from MEXT (the Ministry of Education, Culture, Sports, Science and Tech- nology of Japan). This work is also financially supported by the Young Research Overseas Visits Program for Vitalizing Brain Circulation (R2211) and the Institutional Program for Young Researcher Overseas Visits (R29) by JSPS (Japan Society for the Promotion of Science) as well as the JSPS core-to-core program (No. 17004). We also acknowledge the support of the Mitsubishi Foundation and the Sumitomo Foundation. This research was supported by the grant-in-aid for Nagoya University Global COE Program, "Quest for Fundamental Principles in the Universe: From Particles to the Solar System and the Cosmos," from MEXT. The satellite internet connection for NANTEN2 was provided by the Australian Research Council.

\section{APPENDIX A \\ VELOCITY CHANNEL DISTRIBUTIONS IN RX J1713.7-3946}

We show velocity channel distributions of ${ }^{12} \mathrm{CO}(J=1-0$, 2-1) and $\mathrm{HI}_{\mathrm{I}}$ every $1 \mathrm{~km} \mathrm{~s}^{-1}$ from $-20 \mathrm{~km} \mathrm{~s}^{-1}$ to $0 \mathrm{~km} \mathrm{~s}^{-1}$ superposed on the $\mathrm{TeV} \gamma$-ray distribution in Figure 11.

\section{APPENDIX B}

\section{EXPANDING MOTION OF THE DARK H I SE CLOUD}

Figure 12 (left) shows schematically an expanding spherical shell of radius $R_{0}=9 \mathrm{pc}$ and uniform expansion velocity $V_{0}=$ $10 \mathrm{~km} \mathrm{~s}^{-1}$, and Figure 12 (right) a position-velocity diagram of the shell, where the ellipsoidal nature of the shell is not taken into account for simplicity. Figure 13 shows three representative velocity channel distributions of the dark $\mathrm{H}$ I SE cloud for a velocity range from -20 to $-10 \mathrm{~km} \mathrm{~s}^{-1}$ and shows that the SE cloud is extended to the north. The extension shifts toward the northwest with a velocity decrease from -10 to $-20 \mathrm{~km} \mathrm{~s}^{-1}$ as is consistent with the iso-velocity contours expected from the shell model in Figure 12. Figure 14 shows another presentation of the kinematical details of the SE cloud in position-velocity diagrams. We choose a line, AB, passing through the center of the SNR and the SE cloud, and another line, CD, passing through the SE cloud in the north-south direction (Figure 14(a)). We show a position-velocity distribution of $\mathrm{H}$ I along the line AB (Figure 14(b)) and H i profiles along the two lines AB and CD (Figure 14(c)). We find that the SE cloud is extended to the northwest with a large velocity gradient of $10 \mathrm{~km} \mathrm{~s}^{-1}$ per 0.5 , or $\sim 1.2 \mathrm{~km} \mathrm{~s}^{-1} \mathrm{pc}^{-1}$. The H I profiles in Figure 14(c) show that the dips are deep and clear at $b$, less than -0.5 , but become shallower above $b=-0.5$. The shallower dips make it nontrivial to quantify the dips at $b$ higher than -0.5 ; we note that, even when the dips are not clearly seen, the H I probably suffers from self-absorption to some extent as suggested by the weaker $\mathrm{H}_{\mathrm{I}}$ brightness at $-12 \mathrm{~km} \mathrm{~s}^{-1}$ toward $b=-0.52$ than toward $b=$ -0.35 (line $\mathrm{AB}$ ). We note that the strong velocity gradient in Figures 13 and 14 is consistent with the blueshifted part of an expanding shell. The strong velocity gradient is interpreted in terms of the expanding shell as depicted by a white circle in the position-velocity diagram (Figure 14(b)). The blueshift by $10 \mathrm{~km} \mathrm{~s}^{-1}$ toward the center of the SNR indicates that this part of the shell is in the foreground. This is consistent with the dips being due to self-absorption against the background $\mathrm{H}_{\mathrm{I}}$ emission. We also infer that the swept-up shell is highly nonuniform since the broad $\mathrm{H}$ I dips are seen only in a quarter of the shell. 

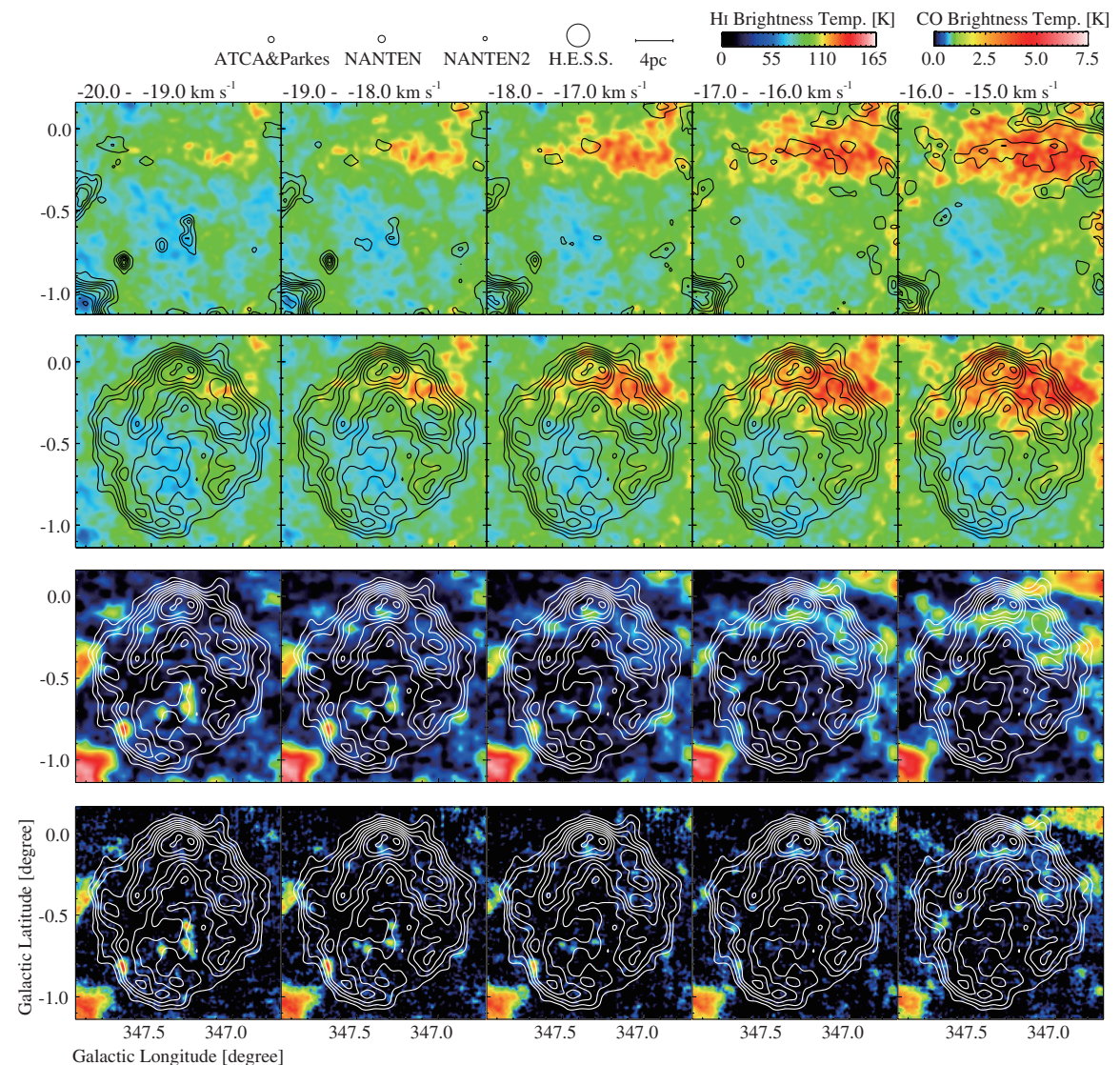

Galactic Longitude [degree]

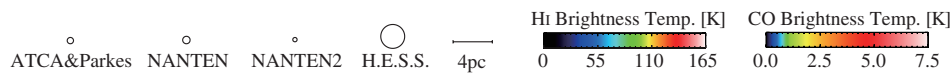
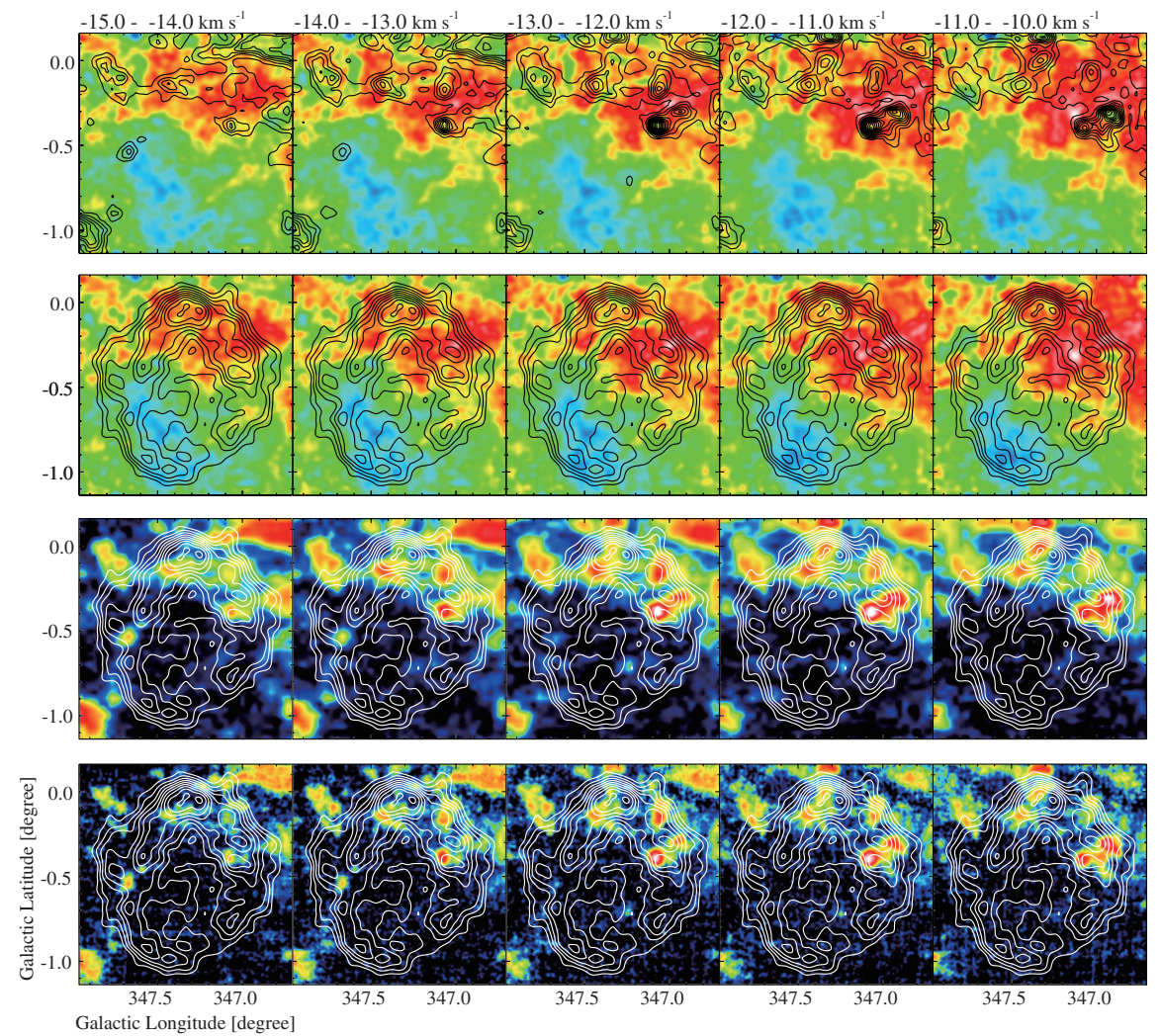

Figure 11. Velocity channel distributions of ${ }^{12} \mathrm{CO}(J=1-0,2-1)$ emission and $\mathrm{H}$ I brightness temperature overlaid on the TeV $\gamma$-ray distribution. First-row panels (top): $\mathrm{H}$ I image and ${ }^{12} \mathrm{CO}(J=1-0)$ contours. Second-row panels: $\mathrm{H}$ I image superposed on the $\mathrm{TeV} \gamma$-ray contours. Third-row panels: ${ }^{12} \mathrm{CO}(J=1-0)$ image superposed on the $\mathrm{TeV} \gamma$-ray contours. Fourth-row panels: ${ }^{12} \mathrm{CO}(J=2-1)$ image superposed on the $\mathrm{TeV} \gamma$-ray contours. Each panel shows $\mathrm{CO}$ and $\mathrm{H}$ I distributions every $1 \mathrm{~km} \mathrm{~s}^{-1}$ in a velocity range from -20 to $0 \mathrm{~km} \mathrm{~s}^{-1}$. The lowest contour levels of $\mathrm{CO}$ and $\mathrm{TeV} \gamma$-rays are $0.73 \mathrm{~K}(\sim 3 \sigma)$ and 20 smoothed counts, and contour intervals of CO and $\mathrm{TeV} \gamma$-rays are $0.73 \mathrm{~K}(\sim 3 \sigma)$ and 10 smoothed counts, respectively. 

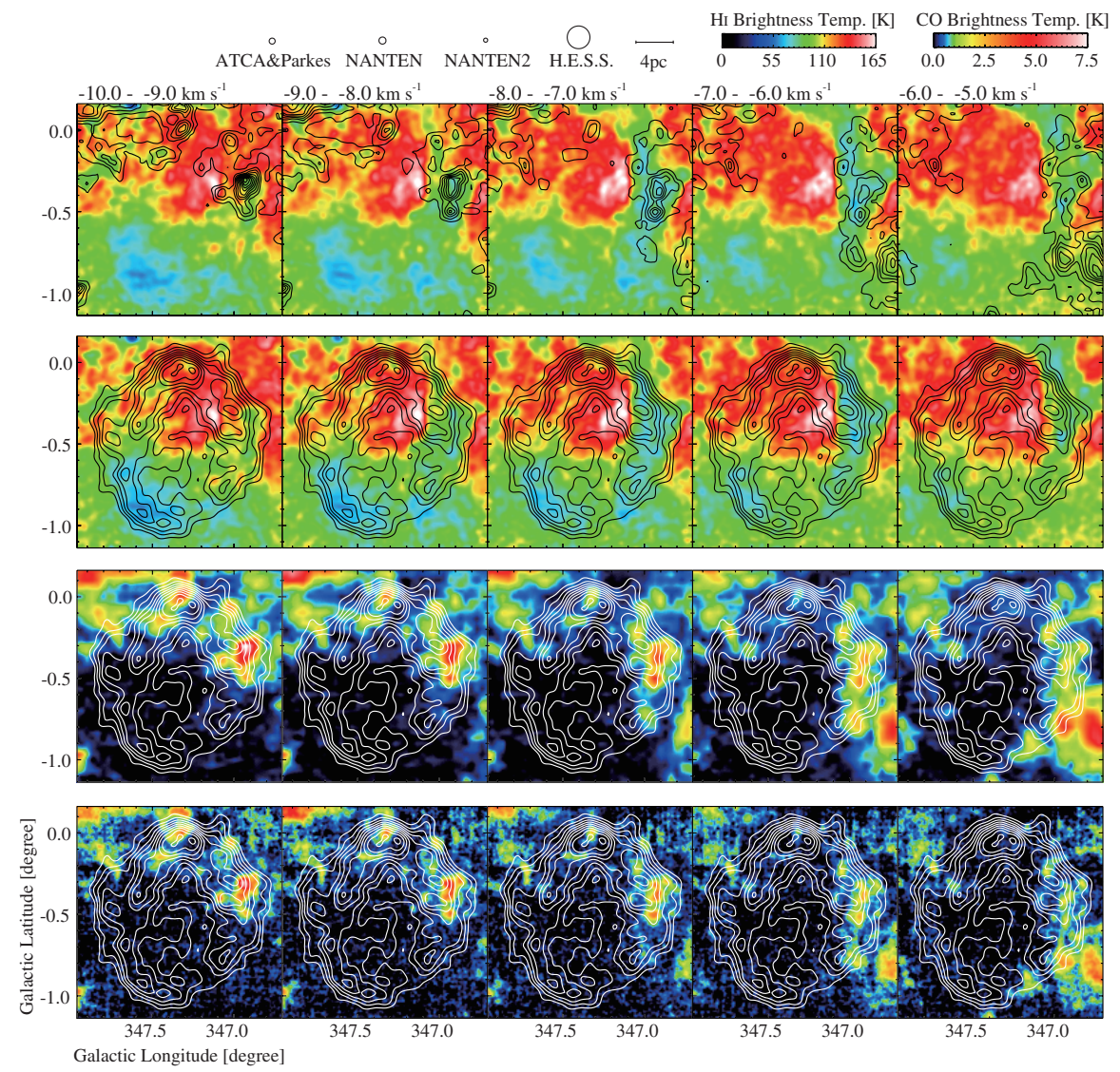

Galactic Longitude [degree]
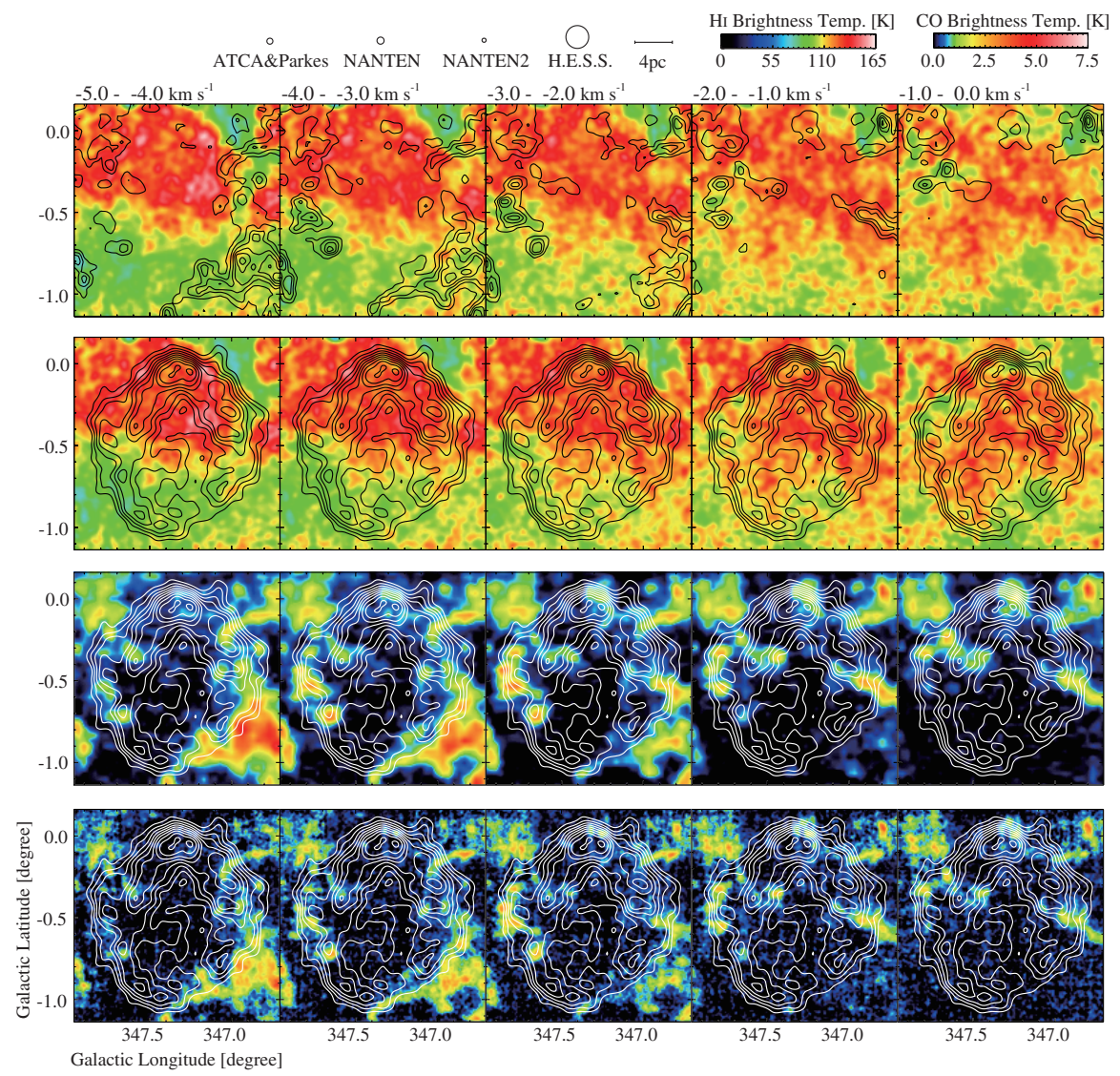

Figure 11. (Continued) 

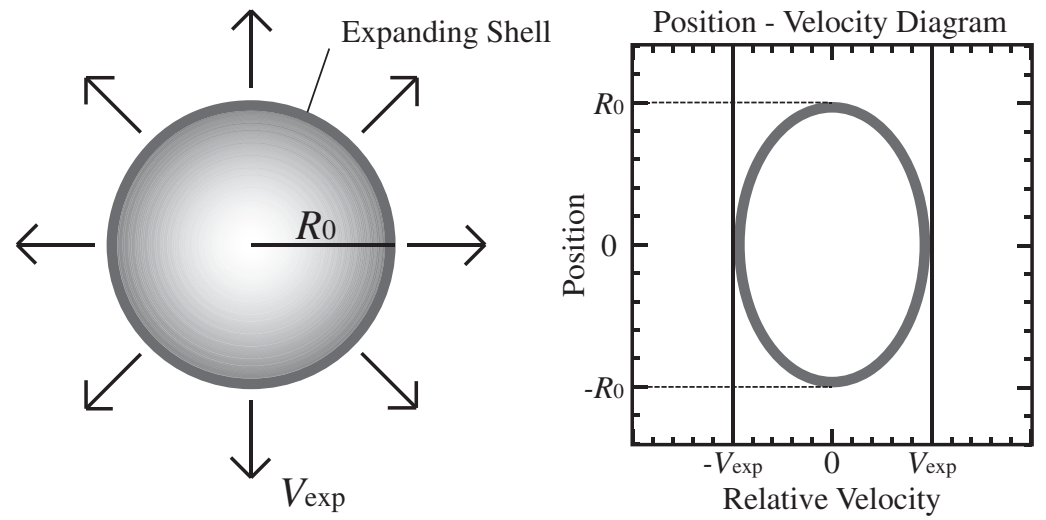

Figure 12. Schematic image of a uniformly expanding shell and its velocity distribution in the position-velocity plane. Here, we assume a radius of the shell $R_{0}$ and an expansion velocity $V_{\exp }$ of $9 \mathrm{pc}$ and $10 \mathrm{~km} \mathrm{~s}^{-1}$, respectively.
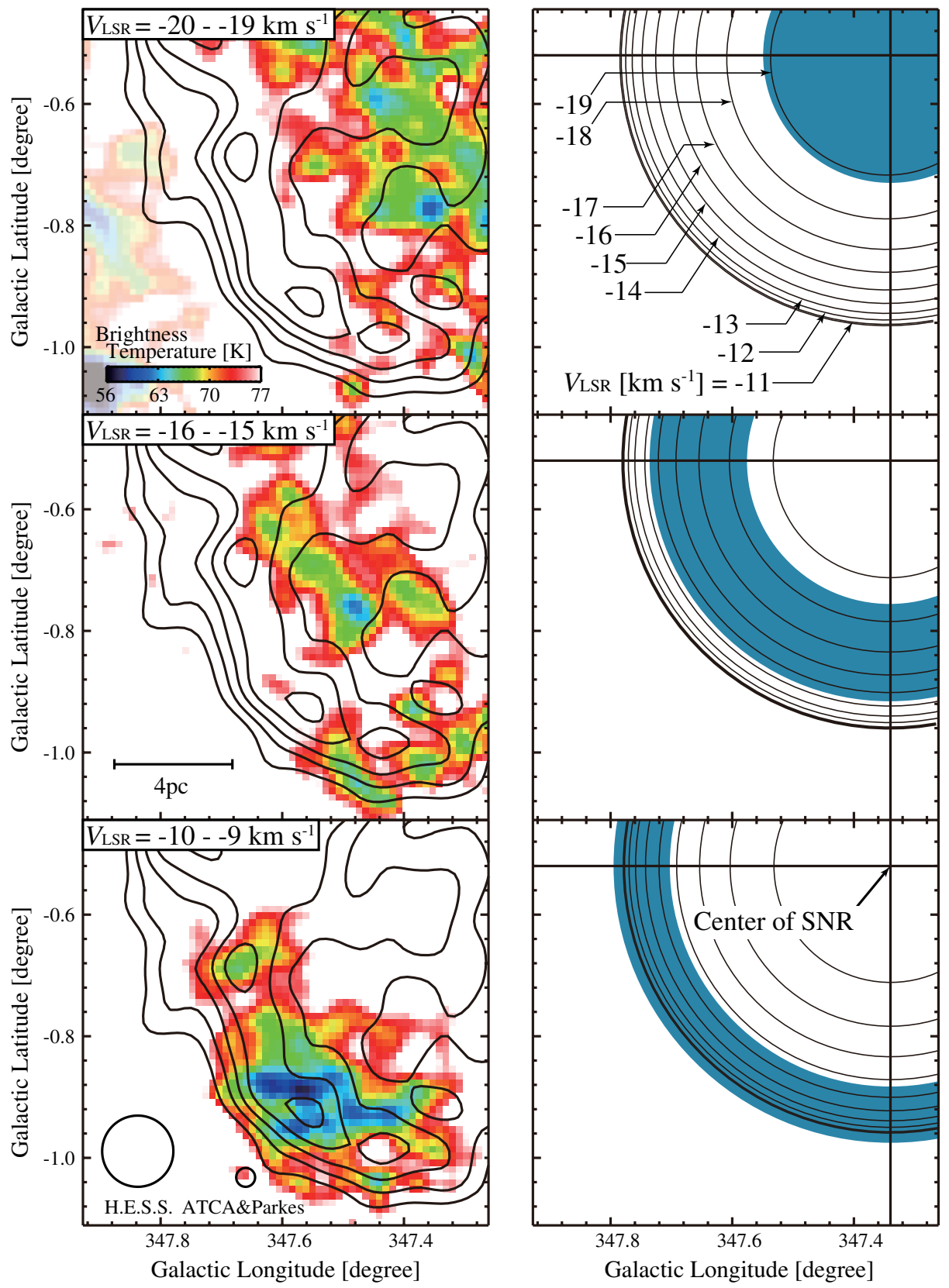

Figure 13. Left: velocity channel distributions of $\mathrm{H}$ I averaged brightness temperature toward the $\mathrm{SE}$ cloud superposed on the $\mathrm{TeV} \gamma$-ray contours. TeV $\gamma$-ray contours are plotted every 10 smoothed counts from 20 smoothed counts. The faded area in the upper panel is a component unrelated to the SNR. Right: model velocity distributions of an expanding shell shown in Figure 12. Iso-velocity lines are shown here, and blue areas show the corresponding velocity range shown in the left panels. 

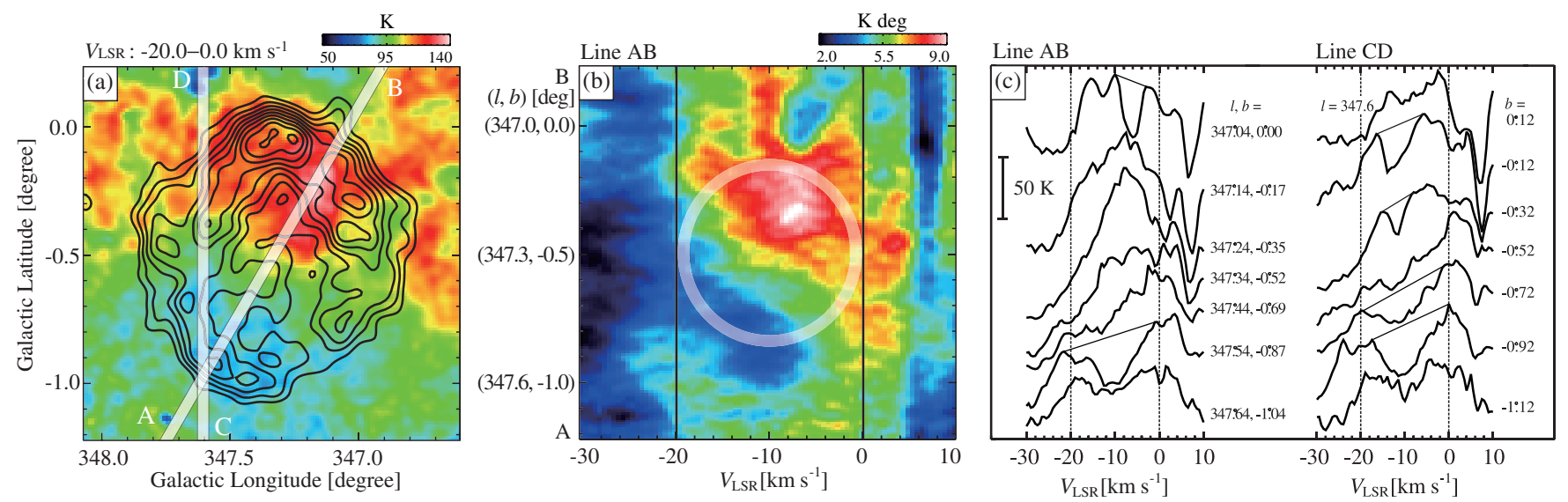

Figure 14. (a) Averaged brightness temperature distribution of $\mathrm{H}$ in a velocity range from -20 to $0 \mathrm{~km} \mathrm{~s}^{-1}$. Contours show the H.E.S.S. TeV $\gamma$-rays (Aharonian et al. 2007) and are plotted every 10 smoothed counts from 10 smoothed counts. The line $\mathrm{AB}$ is inclined by $60^{\circ}$ to the Galactic plane and the line $\mathrm{CD}$ passes the center of the SNR. (b) Position-velocity distribution of $\mathrm{H}$ I along the line $\mathrm{AB}$ in (a). The velocity resolution is smoothed to $1 \mathrm{~km} \mathrm{~s}^{-1}$ and the integration interval is $200^{\prime \prime}$. The white circle shows a schematic image of an expanding spherical shell (Figure 12). (c) H I spectra along the lines AB and CD in (a). Expected profiles of H I self-absorption are shown by straight lines in the spectra with significant H I dips.
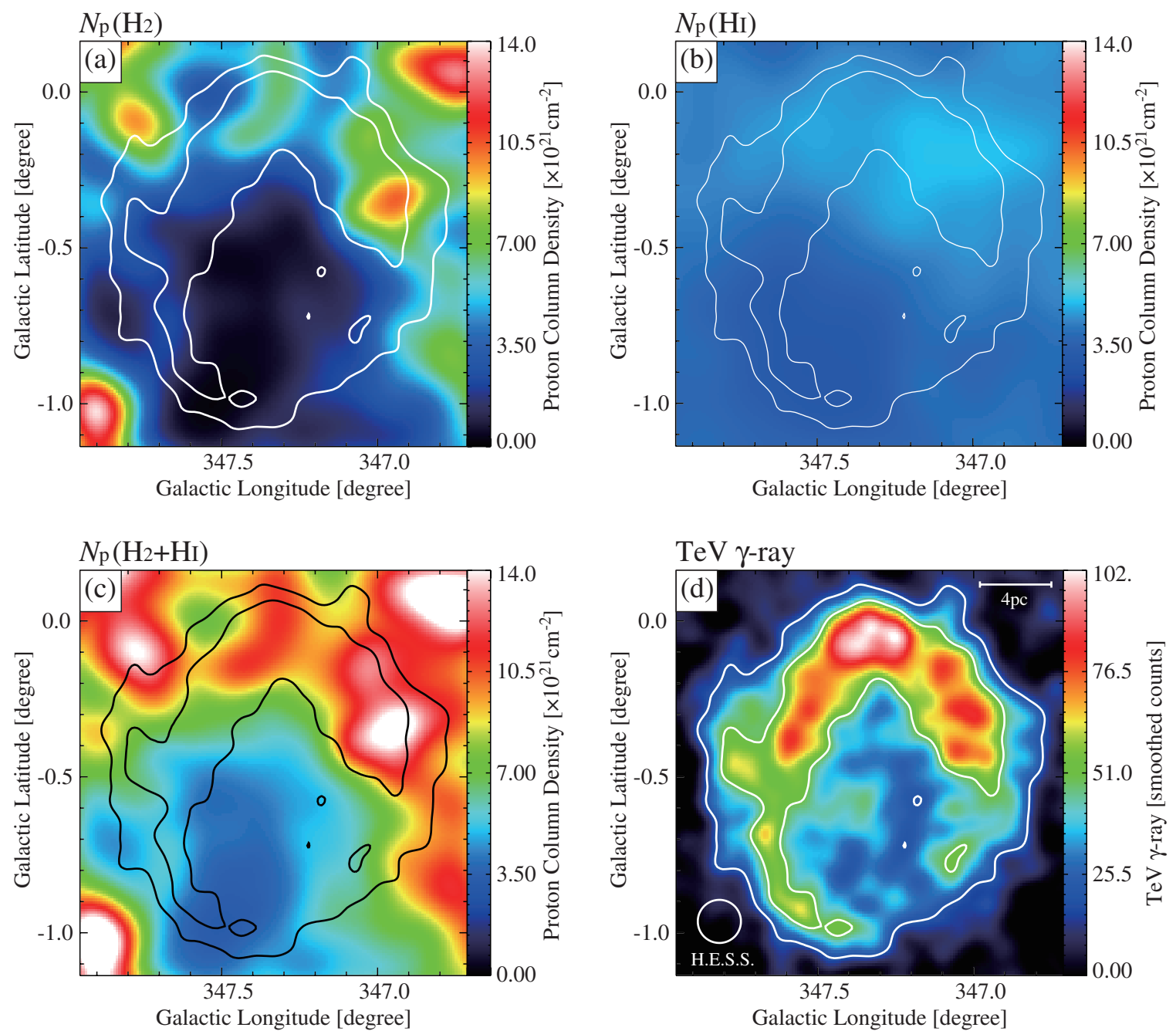

Figure 15. (a-c) Distributions of ISM proton column density, $N_{\mathrm{p}}$, are shown. All the data sets used for the three images are smoothed to have an HPBW of 8.3 , the same figure as the HPBW of H.E.S.S. (a) $N_{\mathrm{p}}\left(\mathrm{H}_{2}\right)$ estimated from ${ }^{12} \mathrm{CO}(J=1-0)$. (b) $N_{\mathrm{p}}(\mathrm{HI})$ estimated from H I with the correction of self-absorption. Here, we assume for reference that the $\mathrm{H}$ I emission is optically thin and the $\mathrm{H}$ I self-absorption is not taken into account. (c) $N_{\mathrm{p}}\left(\mathrm{H}_{2}+\mathrm{H}_{\mathrm{I}}\right)$ estimated by summing up of $N_{\mathrm{p}}\left(\mathrm{H}_{2}\right)$ and $N_{\mathrm{p}}(\mathrm{H} \mathrm{I})$. (d) $\mathrm{TeV} \gamma$-ray distribution. Contours are plotted every 50 smoothed counts from 20 smoothed counts. 

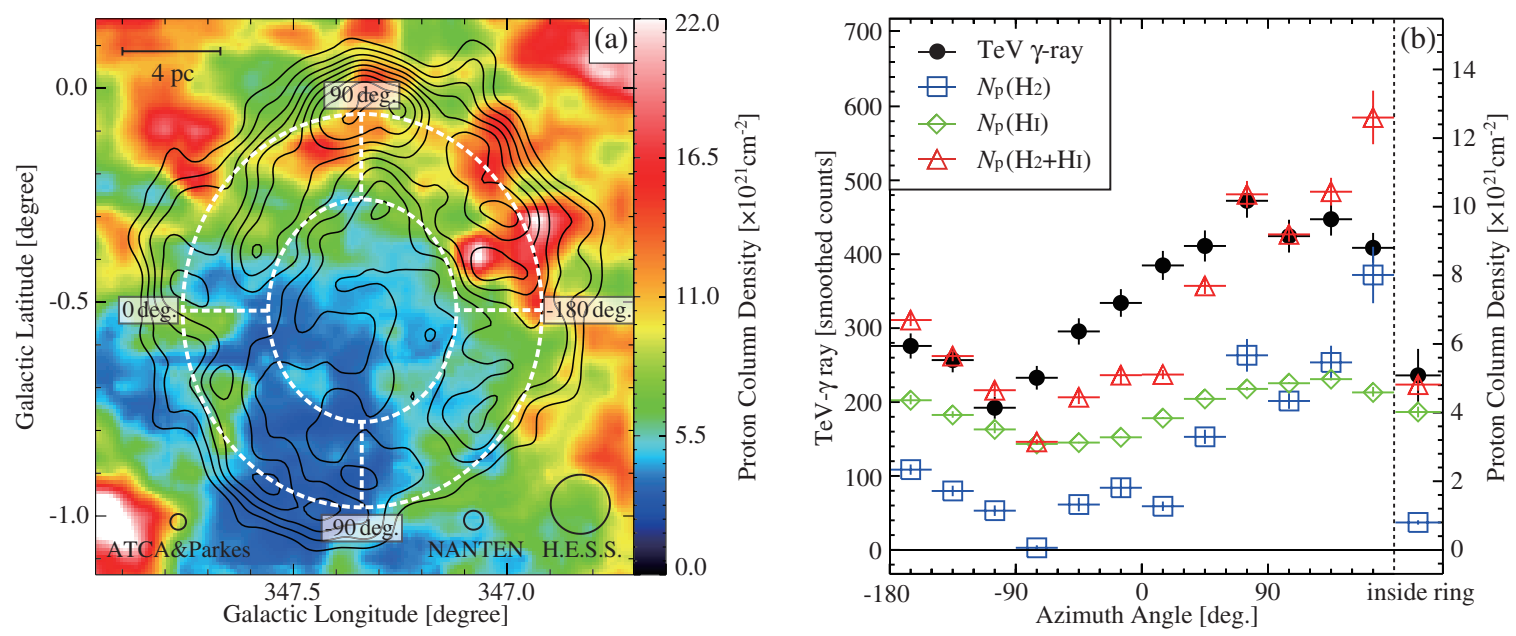

Figure 16. (a) Distribution of column density of ISM protons $N_{\mathrm{p}}\left(\mathrm{H}_{2}+\mathrm{HI}\right)$ in a velocity range from -20 to $0 \mathrm{~km} \mathrm{~s}^{-1}$, where the $\mathrm{H}_{\mathrm{I}}$ is assumed to be optically thin and without self-absorption. Contours and two elliptical rings are the same as in Figure 8(a). (b) Azimuthal distributions of $N_{\mathrm{p}}\left(\mathrm{H}_{2}\right), N_{\mathrm{p}}\left(\mathrm{H}_{\mathrm{I}}\right), N_{\mathrm{p}}\left(\mathrm{H}_{2}+\mathrm{HI}\right)$, and TeV $\gamma$-ray smoothed counts per beam in the two elliptical rings in (a). The same plots inside of the inner ring are shown on the right side in (b).

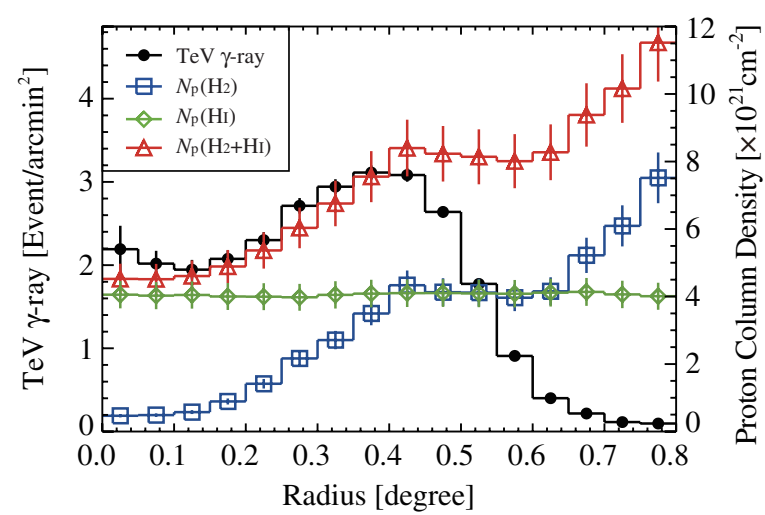

Figure 17. Radial distributions of averaged values of TeV $\gamma$-rays, $N_{\mathrm{p}}\left(\mathrm{H}_{2}\right)$, $N_{\mathrm{p}}(\mathrm{HI})$, and $N_{\mathrm{p}}\left(\mathrm{H}_{2}+\mathrm{HI}\right)$, where the $\mathrm{HI}_{\mathrm{I}}$ is assumed to be optically thin as in Figure 15. $N_{\mathrm{p}}\left(\mathrm{H}_{2}\right)$ and $N_{\mathrm{p}}(\mathrm{HI})$ show column densities estimated from ${ }^{12} \mathrm{CO}(J=$ $1-0)$ and $\mathrm{HI}$, respectively, and $N_{\mathrm{p}}\left(\mathrm{H}_{2}+\mathrm{H} \mathrm{I}\right)$ shows the total ISM column density, the sum of $N_{\mathrm{p}}\left(\mathrm{H}_{2}\right)$ and $N_{\mathrm{p}}(\mathrm{HI})$.

\section{APPENDIX C}

\section{ANALYSIS OF THE H I EMISSION: THE OPTICALLY THIN CASE}

The present analysis has shown that the $\mathrm{HI}$ is self-absorbed in part of the SNR as indicated by the HI dips, and the $\mathrm{HI}$ column density is estimated by taking into account the selfabsorption (Figure 7). In order to see the effects of the selfabsorption quantitatively, here we show for comparison the ISM proton distribution in the optically thin case, which does not take into account the self-absorption. Figure 15, equivalent to the self-absorption case in Figure 7, includes the H I column density distribution for the optically thin assumption smoothed to the H.E.S.S. resolution ((b) and (c)), where the SE cloud is not seen. Figures 15(a) and (d) are the same with those in Figure 7. Figure 16 is equivalent to Figure 8. Figure 16(a) is the total ISM proton column density for the optically thin H I at NANTEN resolution overlaid on the $\mathrm{TeV} \gamma$-ray distribution. Figure 16(b) is the corresponding azimuthal distribution of ISM protons and $\mathrm{TeV} \gamma$-rays, where the ISM protons are deficient in azimuthal angle from $-90^{\circ}$ to $0^{\circ}$ compared to Figure 8(b). Figure 17 is equivalent to Figure 10, and shows the radial distribution of ISM protons for the optically thin $\mathrm{H}$ I without correction for the $\mathrm{HI}$ self-absorption. In the smoothed radial distribution, the effect of the self-absorption is not so obvious.

\section{REFERENCES}

Abdo, A. A., Ackermann, M., Ajello, M., et al. 2010, Science, 327, 1103 Abdo, A. A., Ackermann, M., Ajello, M., et al. 2011, ApJ, 734, 28 Acero, F., Ballet, J., Decourchelle, A., et al. 2009, A\&A, 505, 157 Ade, P. A. R., Aghanim, N., Arnaud, M., et al. 2011, arXiv:1101.2029 Aharonian, F. A., Akhperjanian, A. G., Aye, K.-M., et al. 2004, Nature, 432, 75 Aharonian, F., Akhperjanian, A. G., Bazer-Bachi, A. R., et al. 2006a, ApJ, 636, 777

Aharonian, F., Akhperjanian, A. G., Bazer-Bachi, A. R., et al. 2006b, A\&A, 449,223

Aharonian, F., Akhperjanian, A. G., Bazer-Bachi, A. R., et al. 2007, A\&A, 464 235

Allen, M., \& Robinson, G. W. 1977, ApJ, 212, 396

Bell, A. R. 1978, MNRAS, 182, 147

Berezhko, E. G., \& Völk, H. J. 2008, A\&A, 492, 695

Bertsch, D. L., Dame, T. M., Fichtel, C. E., et al. 1993, ApJ, 416, 587

Blandford, R. D., \& Ostriker, J. P. 1978, ApJ, 221, L29

Casanova, S., Aharonian, F. A., Fukui, Y., et al. 2010a, PASJ, 62, 769

Casanova, S., Jones, D. I., Aharonian, F. A., et al. 2010b, PASJ, 62, 1127

Cassam-Chenaï, G., Decourchelle, A., Ballet, J., et al. 2004, A\&A, 427, 199

Dickey, J. M., \& Lockman, F. J. 1990, ARA\&A, 28, 215

Dobashi, K., Uehara, H., Kandori, R., et al. 2005, PASJ, 57, 1

Ellison, D. C., Patnaude, D. J., Slane, P., \& Raymond, J. 2010, ApJ, 712, 287

Ellison, D. C., \& Vladimirov, A. 2008, ApJ, 673, L47

Enomoto, R., Tanimori, T., Naito, T., et al. 2002, Nature, 416, 823

Fang, J., Tang, Y., \& Zhang, L. 2011, ApJ, 731, 32

Fukui, Y. 2008, in AIP Conf. Proc. 1085, Proc. of 4th International Meeting on

High-Energy Gamma-Ray Astronomy, ed. F. A. Aharonian, W. Hofmann, \&

F. Rieger (Melville, NY: AIP), 104

Fukui, Y., \& Kawamura, A. 2010, ARA\&A, 48, 547

Fukui, Y., Moriguchi, Y., Tamura, K., et al. 2003, PASJ, 55, 61

Gabici, S., Aharonian, F. A., \& Blasi, P. 2007, Ap\&SS, 309, 365

Gabici, S., Aharonian, F. A., \& Casanova, S. 2009, MNRAS, 396, 1629

Giuliani, A., Tavani, M., Bulgarelli, A., et al. 2010, A\&A, 516, L11

Goldsmith, P. F., Li, D., \& Krčo, M. 2007, ApJ, 654, 273

Grenier, I. A., Casandjian, J.-M., \& Terrier, R. 2005, Science, 307, 1292

Inoue, T., Yamazaki, R., \& Inutsuka, S. 2009, ApJ, 695, 825

Inoue, T., Yamazaki, R., Inutsuka, S., \& Fukui, Y. 2012, ApJ, 744, 71

Jenkins, E. B., \& Savage, B. D. 1974, ApJ, 187, 243

Jones, F. C., \& Ellison, D. C. 1991, Space Sci. Rev., 58, 259

Jun, B.-I., \& Norman, M. L. 1996, ApJ, 465, 800

Katz, B., \& Waxman, E. 2008, J. Cosmol. Astropart. Phys., JCAP01(2008)018

Koyama, K., Kinugasa, K., Matsuzaki, K., et al. 1997, PASJ, 49, 7

Krčo, M., \& Goldsmith, P. F. 2010, ApJ, 724, 1402

Lazendic, J. S., Slane, P. O., Gaensler, B. M., et al. 2004, ApJ, 602, 271

Malkov, M. A., \& O’C Drury, L. 2001, Rep. Prog. Phys., 64, 429

Matsunaga, K., Mizuno, N., Moriguchi, Y., et al. 2001, PASJ, 53, 1003 
McClure-Griffiths, N. M., Dickey, J. M., Gaensler, B. M., et al. 2005, ApJS, 158,178

Moriguchi, Y., Tamura, K., Tawara, Y., et al. 2005, ApJ, 631, 947

Morlino, G., Amato, E., \& Blasi, P. 2009, MNRAS, 392, 240

Ohama, A., Dawson, J. R., Furukawa, N., et al. 2010, ApJ, 709, 975

Patnaude, D. J., Slane, P., Raymond, J. C., \& Ellison, D. C. 2010, ApJ, 725 1476

Pfeffermann, E., \& Aschenbach, B. 1996, in Proc. Röntgenstrahlung from the Universe, ed. H. U. Zimmermann, J. Trümper, \& H. Yorke (MPE Rep. 263; Garching: MPE), 267

Porter, T. A., Moskalenko, I. V., \& Strong, A. W. 2006, ApJ, 648, L29

Sano, H., Sato, J., Horachi, H., et al. 2010, ApJ, 724, 59
Sato, F., \& Fukui, Y. 1978, AJ, 83, 1607

Slane, P., Gaensler, B. M., Dame, T. M., et al. 1999, ApJ, 525, 357

Tanaka, T., Uchiyama, Y., Aharonian, F. A., et al. 2008, ApJ, 685, 988

Torii, K., Enokiya, R., Sano, H., et al. 2011, ApJ, 738, 46

Uchiyama, Y., Aharonian, F. A., \& Takahashi, T. 2003, A\&A, 400, 567

Uchiyama, Y., Aharonian, F. A., Tanaka, T., Takahashi, T., \& Maeda, Y. 2007, Nature, 449, 576

Wang, Z. R., Qu, Q.-Y., \& Chen, Y. 1997, A\&A, 318, 59

Yamamoto, H., Kawamura, A., Tachihara, K., et al. 2006, ApJ, 642, 307

Zirakashvili, V. N., \& Aharonian, F. A. 2007, A\&A, 465, 695

Zirakashvili, V. N., \& Aharonian, F. A. 2010, ApJ, 708, 965 